\title{
Constraining the mass-richness relationship of redMaPPer clusters with angular clustering
}

\author{
Eric J. Baxter, ${ }^{1 \star}$ Eduardo Rozo, ${ }^{2}$ Bhuvnesh Jain, ${ }^{1}$ Eli Rykoff ${ }^{3,4}$ \\ and Risa H. Wechsler ${ }^{3,4}$ \\ ${ }^{1}$ Center for Particle Cosmology, Department of Physics, University of Pennsylvania, Philadelphia, PA 19104, USA \\ ${ }^{2}$ Department of Physics, University of Arizona, Tucson, AZ 85721, USA \\ ${ }^{3}$ Kavli Institute for Particle Astrophysics and Cosmology, Department of Physics, Stanford University, Stanford, CA 94305, USA \\ ${ }^{4}$ SLAC National Accelerator Laboratory, Menlo Park, CA 94025, USA
}

Accepted 2016 August 1. Received 2016 July 29; in original form 2016 April 11

\begin{abstract}
The potential of using cluster clustering for calibrating the mass-richness relation of galaxy clusters has been recognized theoretically for over a decade. Here, we demonstrate the feasibility of this technique to achieve high-precision mass calibration using redMaPPer clusters in the Sloan Digital Sky Survey North Galactic Cap. By including cross-correlations between several richness bins in our analysis, we significantly improve the statistical precision of our mass constraints. The amplitude of the mass-richness relation is constrained to 7 percent statistical precision by our analysis. However, the error budget is systematics dominated, reaching a 19 per cent total error that is dominated by theoretical uncertainty in the bias-mass relation for dark matter haloes. We confirm the result from Miyatake et al. that the clustering amplitude of redMaPPer clusters depends on galaxy concentration as defined therein, and we provide additional evidence that this dependence cannot be sourced by mass dependences: some other effect must account for the observed variation in clustering amplitude with galaxy concentration. Assuming that the observed dependence of redMaPPer clustering on galaxy concentration is a form of assembly bias, we find that such effects introduce a systematic error on the amplitude of the mass-richness relation that is comparable to the error bar from statistical noise. The results presented here demonstrate the power of cluster clustering for mass calibration and cosmology provided the current theoretical systematics can be ameliorated.
\end{abstract}

Key words: methods: analytical-cosmology: observations-large-scale structure of Universe.

\section{INTRODUCTION}

The abundance of galaxy clusters is a powerful probe of cosmology (Weinberg et al. 2013). The observed number density of clusters as a function of redshift, $n(z)$, is sensitive to both the expansion history of the Universe and to the growth of structure. This dual sensitivity enables cluster abundance measurements to distinguish between dark energy models and modified gravity (Huterer et al. 2015). However, modelling the cluster abundance to extract cosmological constraints is challenging because the abundance depends sensitively on the masses of the observed clusters. Because clusters live at the extreme tails of the mass function where the abundance is falling exponentially, small changes in the cluster mass can have a large impact on the predicted cluster abundance. Moreover, measuring cluster masses is difficult because it requires

^E-mail: ebax@sas.upenn.edu relating observable quantities to cluster mass. The mass-observable relationships are often noisy and subject to large systematic uncertainties (Rozo et al. 2014; Sereno \& Ettori 2015). For these reasons, the dominant systematic currently affecting cosmological constraints derived from cluster abundance measurements is uncertainty in cluster masses (Vikhlinin et al. 2009; Rozo et al. 2010; Sehgal et al. 2011; Benson et al. 2013; Mantz et al. 2015; Planck Collaboration XXIV 2015a). If these mass uncertainties can be reduced, the full power of cluster abundance measurements can be exploited, and clusters will become highly competitive probes of dark energy and modified gravity (Albrecht et al. 2006).

The spatial clustering of galaxy clusters is sensitive to cluster mass, with higher mass clusters being more strongly clustered on the sky. To quantify the degree of clustering, it is common to define the bias as the square root of the ratio of the correlation function of clusters - which we identify with massive dark matter haloes to that of all matter. The relationship between halo bias and halo mass, $b(M)$, is well understood via the halo model (for a review 
see Cooray \& Sheth 2002) and can be calibrated from simulations (e.g. Tinker et al. 2010). Several authors have highlighted the possibility of using measurements of the clustering biases of clusters to do a 'self-calibrated' cluster cosmology analysis, i.e. to jointly fit for cosmological parameters as well as parameters governing the relationship between observable quantities and cluster mass (e.g. Majumdar \& Mohr 2003; Lima \& Hu 2004; Holder \& Kosowsky 2004; Hu \& Cohn 2006).

In this work, we perform the first half of the self-calibration programme, i.e. measuring the clustering biases of a catalogue of optically detected galaxy clusters and using the measured biases to constrain the mass-observable relationship. To this end, we use the catalogue of redMaPPer clusters (Rykoff et al. 2014) identified in the Sloan Digital Sky Survey (SDSS). The relevant observable for the redMaPPer clusters is the richness, a measure of the number of galaxies within the cluster. Using the measured correlation functions and the predicted $b(M)$, we calibrate the mass-richness relation for the redMaPPer clusters.

Two of the most significant challenges for using cluster clustering to calibrate cluster masses are line-of-sight cluster projections and assembly bias. Chance alignment of clusters along the line of sight can alter the richness estimates of these clusters and change the measured clustering amplitude. If this effect is not accounted for, constraints on the mass-richness relation of the redMaPPer clusters could be biased. Assembly bias, on the other hand, refers to the dependence of halo clustering on any halo properties other than halo mass, such as assembly history or concentration (Gao, Springel \& White 2005; Wechsler et al. 2006; Jing, Suto \& Mo 2007; Dalal et al. 2008). Assembly bias complicates any attempt to extract constraints on the mass-richness relation or cosmology using $b(M)$ alone. Below, we develop methodology to account for the effects of both cluster projections and assembly bias on our constraints on the parameters of the mass-richness relationship.

The outline of the paper is as follows: we introduce the formalism for describing the correlation function measurements in Section 2; the cluster catalogue is described in Section 3; the measurement of the correlation function and its covariance are described in Section 4; our model for the correlation function and fitting procedure - including our treatment of projection and assembly bias effects - are described in Section 5; results are presented in Section 6; we explore the various contributions to our error budget in Section 7; the cosmology dependence of our constraints is considered in Section 8; finally, our conclusions are given in Section 9.

Throughout, all cluster masses refer to $M_{200 m}$, i.e. the mass enclosed within a sphere centred on a cluster such that the mean density within that sphere is 200 times the mean density of the Universe at the cluster's redshift. Our fiducial analysis assumes the best-fitting flat $\Lambda$ cold dark matter $(\Lambda \mathrm{CDM})$ model from an analysis of cosmic microwave background data and other data sets by Planck Collaboration XIII (2015b). The cosmological parameters in this model are $\Omega_{\mathrm{m}}=0.309, h_{0}=H_{0} /\left(100 \mathrm{~km} \mathrm{~s}^{-1} \mathrm{Mpc}^{-1}\right)=0.677, \Omega_{\mathrm{b}}=0.0486$, $\tau=0.066, n_{\mathrm{s}}=0.9667$ and $A_{\mathrm{s}}=2.14 \times 10^{-9}$ at a pivot scale of $k=0.05 \mathrm{Mpc}^{-1}$. In Section 8, we explore how our parameter constraints are affected by variations in the assumed cosmological model.

\section{FORMALISM}

We define $n(\hat{\phi})$ to be the projected density of clusters in the direction specified by the unit vector $\hat{\phi}$. The overdensity in the same direction is then defined as

$\delta(\hat{\phi}) \equiv \frac{n(\hat{\phi})-\bar{n}}{\bar{n}}$,

where $\bar{n}$ is the average of $n(\hat{\phi})$ over all $\hat{\phi}$. The clustering of clusters on the sky can be characterized in terms of the angular correlation function, $w(\theta)$ :

$w(\theta)=\left\langle\delta(\hat{\phi}) \delta\left(\hat{\phi}^{\prime}\right)\right\rangle$,

where the average is taken over all possible $\hat{\phi}$ and $\hat{\phi}^{\prime}$ such that the angular separation between $\hat{\phi}$ and $\hat{\phi}^{\prime}$ is $\theta$.

We measure the angular correlation function of the redMaPPer clusters in bins of richness and redshift; in addition, we also measure several cross-bin angular correlations. To keep the notation simple, we use a single Greek superscript to represent both the richness and redshift bins. The correlation function between richness/redshift bin $\alpha$ and richness/redshift bin $\beta$ will be denoted with $w^{\alpha \beta}(\theta)$. Occasionally, we suppress the superscripts on $w(\theta)$ for notational convenience.

The correlation function $w^{\alpha \beta}(\theta)$ is related to the angular power spectrum, $C_{\ell}^{\alpha \beta}$, by

$w^{\alpha \beta}(\theta)=\sum_{l=0}^{\infty}\left(\frac{2 l+1}{4 \pi}\right) P_{\ell}(\cos \theta) C_{\ell}^{\alpha \beta}$,

where $P_{\ell}$ is the Legendre polynomial of order $\ell$. We can relate $C_{\ell}^{\alpha \beta}$ for redMaPPer clusters to the matter power spectrum using the Limber approximation

$C_{\ell}^{\alpha \beta}=\int \mathrm{d} z W^{\alpha}(z) W^{\beta}(z) \frac{H(z)}{d_{A}^{2}(z)} b^{2}(k, z) P\left(k=\frac{\ell+1 / 2}{\chi(z)} ; z\right)$,

where $H(z)$ is the Hubble parameter at redshift $z, d_{\mathrm{A}}(z)$ is the angular diameter distance, $\chi(z)$ is the comoving distance, and we have assumed a spatially flat Universe (Limber 1953). The weight function $W^{\alpha}(z)$ is the distribution of clusters in the $\alpha$ th richness/redshift bin as a function of redshift, normalized such that $\int \mathrm{d} z W^{\alpha}(z)=1$. The $b(z, k)$ term is the bias of the redMaPPer clusters, which we discuss in more detail below. The Limber approximation is expected to be valid when the redshift selection function, $W^{\alpha}(z)$, is much wider than the scales of interest. In our case, the maximum physical scale that we probe is roughly $25 \mathrm{Mpc}$, while the width of the selection function is roughly $280 \mathrm{Mpc}$ (these scales are set by our choice of angular and redshift bins, which are discussed in more detail in Sections 3 and 4). We are therefore safely in the regime for which the Limber approximation is valid.

At large scales, the bias is expected to be independent of $k$. This is the so-called linear bias regime. At small scales, the bias may become scale dependent and is difficult to model. We therefore restrict our analysis to the linear bias regime (but still at scales small enough that the Limber approximation holds). In this limit, the angular correlation function is simply

$w^{\alpha \beta}(\theta)=b^{\alpha} b^{\beta} w_{\mathrm{M}}^{\alpha \beta}(\theta)$,

where $w_{\mathrm{M}}^{\alpha \beta}$ is the matter-matter correlation function, given by equations (3) and (4) with $b(k, z)=1$. The coefficients $b^{\alpha}$ and $b^{\beta}$ are the linear bias parameters for the two richness/redshift bins $\alpha$ and $\beta$; constraining these parameters for the redMaPPer clusters is one of the main goals of this analysis. The bias parameters should be thought of as averages across all clusters in a given richness/redshift bin. 


\section{DATA}

Our analysis uses the redMaPPer (Rykoff et al. 2014) catalogue of clusters identified in the SDSS (York et al. 2000) eighth Data Release (DR8; Aihara et al. 2011). The SDSS DR8 photometric galaxy catalogue includes roughly $14000 \mathrm{deg}^{2}$ of imaging, which was reduced to $\sim 10000 \mathrm{deg}^{2}$ of high-quality contiguous imaging for the purposes of cluster finding using the Baryon Oscillation Spectroscopic Survey mask (Dawson et al. 2013). Given a cluster candidate, redMaPPer uses the SDSS five-band imaging to estimate the probability of any given galaxy in the field of being a red-sequence cluster member of the candidate cluster. The cluster richness is the total number of red-sequence members $\lambda$, and serves as our observable mass proxy. A detailed explanation of the redMaPPer algorithm can be found in Rykoff et al. (2014).

For the main results of this work, we restrict our analysis to redMaPPer clusters identified in the North Galactic Cap (NGC) of SDSS, a contiguous region of $\sim 7000 \mathrm{deg}^{2}$ in the Northern hemisphere. We restrict our analysis to the NGC for two reasons. First, the weak lensing mass calibration of redMaPPer clusters performed by Simet et al. (2016, hereafter S16) is restricted to the NGC (for reasons discussed therein), and imposing the same restriction on our analysis makes comparison between the two works more straightforward. Secondly, as we discuss in Section 7.5, we find some tension between the clustering measurements in the NGC and the Southern Galactic Cap (SGC). We refrain from combining the parameter constraints derived from the NGC and SGC because of this tension, and only present results from the NGC because it represents a larger area than the SGC.

Our analysis relies on the redMaPPer v5.10 catalogue, ${ }^{1}$ an updated version of the original redMaPPer cluster catalogue with a variety of modest improvements. Typical photometric redshift uncertainties are $\sigma_{z} /(1+z) \lesssim 0.01$, with photo $z$ biases controlled at the $\Delta z \approx 0.003$ level. This level of photometric redshift performance is sufficiently high that photometric redshift errors can be safely ignored in our analysis.

We note that while the redMaPPer v5.10 cluster catalogue is publicly available, our analysis relies on a proprietary version of the catalogue that extends the low richness threshold from $\lambda \geq 20$ to $\lambda \geq 5$. As discussed in Rykoff et al. (2014), the $\lambda \geq 20$ threshold is a purposely conservative selection that ensures a clean connection between individual dark matter haloes and galaxy clusters selected by redMaPPer. We demonstrate below why our specific science goal allows us to use low-richness clusters, even if the connection between haloes and galaxy clusters is less secure for these lowrichness systems.

We restrict the catalogue used in this work to clusters with $0.18<z<0.33$. The cut at the high-redshift end ensures that the cluster catalogue is volume limited (Rykoff et al. 2014). The cut at the low-redshift end is chosen to maximize our signal-tonoise while ensuring that we remain in the linear bias regime (see discussion in Section 4).

\section{CORRELATION FUNCTION MEASUREMENT}

We measure $w^{\alpha \beta}(\theta)$ using the pair counting estimator of Landy \& Szalay (1993). The Landy \& Szalay (1993) estimator relies on computing three quantities to estimate the correlation function over a given angular bin: $D D, R R$ and $D R$. The quantity $D D$ ('data-data') is the number of pairs of clusters that have an angular separation that is within the bin, $R R$ ('random-random') is similarly defined for a catalogue of points whose positions have been randomly and independently chosen on the sky and $D R$ ('data-random') is the number of cross-pairs between the cluster and random catalogues. To reduce the effects of statistical noise, the random catalogue is typically generated with $\sim 30-50$ times more points than the data catalogue [although more optimal estimators such as that of Baxter \& Rozo (2013) can reduce this requirement].

For a particular angular bin, the Landy \& Szalay (1993) estimate of the correlation function between richness/redshift bin $\alpha$ and richness/redshift bin $\beta$ is

$$
\begin{aligned}
\hat{w}^{\alpha \beta}= & \left(\frac{D D^{\alpha \beta}}{R R^{\alpha \beta}}\right)\left(\frac{R^{\alpha} R^{\beta}}{D^{\alpha} D^{\beta}}\right)-\left(\frac{D R^{\alpha \beta}}{R R^{\alpha \beta}}\right)\left(\frac{R^{\alpha}}{D^{\alpha}}\right) \\
& -\left(\frac{D R^{\beta \alpha}}{R R^{\alpha \beta}}\right)\left(\frac{R^{\beta}}{D^{\beta}}\right)+1,
\end{aligned}
$$

where $D^{\alpha}$ and $R^{\alpha}$ are the number of data and random points in the $\alpha$ richness/redshift bin, respectively (and similarly for $D^{\beta}$ and $R^{\beta}$ ). The superscript notation indicates which bins are being correlated; $D D^{\alpha \beta}$, for instance, refers to the pair counts between bins $\alpha$ and $\beta$. All pair counting in our analysis is performed using the tree code TREECORR $^{2}$ (Jarvis, Bernstein \& Jain 2004).

In order to account for survey geometry and selection effects, the random catalogue used to compute $D R$ and $R R$ must be restricted to the same sky mask as the data. As the redMaPPer mask is richness and redshift dependent, the creation of an appropriate random catalogue is non-trivial. The random catalogue used in this work is generated as follows: for each cluster in the redMaPPer catalogue, we select a random position in the sky, and test whether the cluster could have been detected at that location. If yes, the cluster is added to the random point catalogue at this random location. If not, that cluster is rejected from the random catalogue. Because of the large number of draws, every cluster is drawn multiple times, and we record the number of times the cluster was added to the random catalogue $\left(N_{1}\right)$, and the number of times the cluster was rejected $\left(N_{2}\right)$. Every cluster is assigned a weight $W=\left(N_{1}+N_{2}\right) / N_{1}$ in the random catalogue to ensure that the weighted random points statistically reproduce the joint richness, redshift and spatial distribution of the parent sample. We modify equation (6) so as to include these weights. Specifically, a random point with weight $W_{i}$ contributes $W_{i}$ to $R$ and $D R$, and two random points $i$ and $j$ contribute a weight $W_{i} W_{j}$ to $R R$.

Our baseline analysis uses $N_{\theta}=4$ angular bins, $N_{\lambda}=4$ richness bins and $N_{z}=2$ redshift bins. We discuss the choice of angular bins in more detail in Section 4.1. The richness bin edges are [5, 20, $28,41, \infty)$, while the redshift bin edges are $[0.18,0.26,0.32]$. The bins have been chosen so that the measurement of $w^{\alpha \beta}(\theta)$ in each richness and redshift bin has roughly the same signal-to-noise.

In addition to measuring the angular auto-correlation functions of the clusters in each richness and redshift bin, we also measure the angular cross-correlations of clusters in the same redshift bin, but in different richness bins. Including the cross-correlations between richness bins considerably enhances the signal-to-noise of our constraints on the clustering bias parameters. The inclusion of the crossrichness bin correlations means that for our baseline analysis, the data vector has dimension $d=N_{\theta} N_{\lambda}\left(N_{\lambda}+1\right) N_{z} / 2=80$. Because $\sigma_{z}$ is small for redMaPPer clusters $\left(\sigma_{z} \lesssim 0.01\right)$ and because the 
redshift bins are wide $(\Delta z \sim 0.07)$, the angular cross-correlations between different redshift bins are expected to be negligible and would therefore contribute little additional information to our bias constraints. Since including these measurements would come at the cost of increasing the size of the covariance matrix - and would therefore make our jackknife covariance estimation less reliable (see below) - we do not include measurements of cross-redshift bin correlations as part of our analysis. We have, however, measured the correlations between redshift bins and have confirmed that they are negligible, as expected. This measurement can be viewed as a check for systematic errors in the redMaPPer catalogue. For instance, if the redMaPPer redshift estimates for some clusters were wrong or if the redMaPPer catalogue were significantly impacted by line-of-sight projections (discussed in more detail in Section 5.2.1), such errors could result in significant angular correlations between different redshift bins.

We compute the covariance matrix of our $w(\theta)$ measurements using a jackknife approach. To estimate the covariance matrix with a jackknife, the survey region is divided into $N_{\mathrm{jk}}$ patches of equal area. The measurement of $w(\theta)$ is repeated with each of the patches removed in turn. We use $\boldsymbol{w}_{i}$ to denote the estimate of $w(\theta)$ with the $i$ th patch removed, where the vector notation indicates that $\boldsymbol{w}_{i}$ includes measurements at several $\theta$ values and for several richness/redshift bins ( $\boldsymbol{w}_{i}$ is assumed to be a column vector). The jackknife covariance matrix estimate is

$\hat{\mathbf{C}}=\frac{N_{\mathrm{jk}}-1}{N_{\mathrm{jk}}} \sum_{i}^{N_{\mathrm{jk}}}\left(\boldsymbol{w}_{i}-\overline{\boldsymbol{w}}\right)\left(\boldsymbol{w}_{i}-\overline{\boldsymbol{w}}\right)^{T}$,

where

$\bar{w}(\theta)=\frac{1}{N_{\mathrm{jk}}} \sum_{i=1}^{N_{\mathrm{jk}}} \boldsymbol{w}_{i}(\theta)$

(see e.g. Norberg et al. 2009). In this work, we use $N_{\mathrm{jk}} \sim 800$ patches, but find that our results are not very sensitive to this choice (see Section 7.6). Since the data vector has 80 elements, there are roughly 10 times as many jackknife patches as the dimension of the covariance matrix.

\subsection{Angular binning}

In principle, since all angular scales contain information about $w(\theta)$, one would like to measure $w(\theta)$ across the widest possible range of $\theta$ to extract the tightest possible constraints on the biases of redMaPPer clusters. At low $\theta$, however, modelling $w(\theta)$ becomes increasingly difficult for two reasons. First, modelling the matter power spectrum at small scales is difficult because of baryonic effects (Jing et al. 2006; van Daalen et al. 2011). Secondly, at small scales, the bias is expected to become scale dependent, and modelling this scale dependence is difficult (see e.g. Hayashi \& White 2008; $\mathrm{Zu}$ et al. 2014, for discussion within the context of halo-mass correlation functions).

To eliminate modelling uncertainties at small angular scales, we restrict our analysis to the angular regime for which the power spectrum can be accurately modelled and the linear bias model is expected to be valid. Imposing this requirement greatly simplifies the analysis as it leaves only one free parameter - the linear bias, $b^{\alpha}$ - to describe the clustering of redMaPPer clusters in the $\alpha$ richness/redshift bin (given a fixed cosmological model).

$\mathrm{Zu}$ et al. (2014) find that a linear bias model can adequately describe the halo-mass correlation function for scales $R \gtrsim 3.5 \mathrm{Mpc}$. Being conservative, we therefore set $\theta_{\min }$ by demanding that the

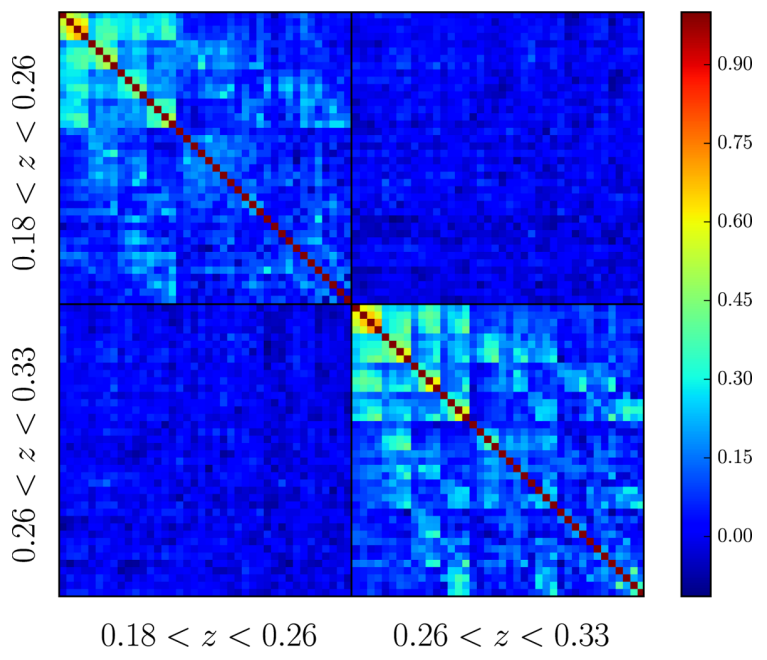

Figure 1. The full correlation matrix $\operatorname{Cov}\left(X_{i}, X_{j}\right) / \sigma_{i} \sigma_{j}$ between each measured $X_{i}=\hat{w}^{\alpha \beta}(\theta)$, computed using a spatial jackknife with roughly 800 subregions. See the text for the ordering of the matrix elements. We find little covariance between the clustering signals of clusters in different redshift bins.

projected distance $R$ spanned by the angle $\theta_{\min }$ be more than a minimum length $R_{\min }=5 h^{-1} \mathrm{Mpc}$ for all clusters in a given redshift bin. This amounts to setting $\theta_{\min } \approx 39$ arcmin for the first redshift bin and $\theta_{\min } \approx 30$ arcmin for the second redshift bin. The boundary of the lowest redshift bin has been chosen to maximize signal-to-noise while maintaining the requirement that $\theta_{\min } d_{\mathrm{A}}\left(z_{\min }\right)=5 \mathrm{~h}^{-1} \mathrm{Mpc}$. We emphasize that the minimum cutoff length is especially conservative in that the cutoff distance is a distance in projection; the true three-dimensional separation is expected to be significantly larger $(\approx 25 \mathrm{Mpc}$ for our cluster sample).

At large scales, modelling the $w^{\alpha \beta}(\theta)$ is straightforward, but computing the covariance of $\hat{w}^{\alpha \beta}(\theta)$ with the jackknife becomes difficult. The jackknife covariance estimate is only expected to yield reliable results for angular scales less than the size of the jackknife patches. For our $N_{\mathrm{jk}} \sim 800$ patches, the typical size of a patch is $\theta_{\text {patch }} \sim 170$ arcmin. We therefore set $\theta_{\max }=100$ arcmin, roughly a factor of 1.7 smaller than the size of the jackknife patches. Our four angular bins are spaced logarithmically between $\theta_{\min }$ and $\theta_{\max }$.

Fig. 1 shows the correlation matrix for the data vector as measured using the jackknife with roughly 800 subregions. The data vector is ordered such that the first 40 elements correspond to the first redshift bin and the last 40 correspond to the second redshift bin. For each redshift bin, the elements are ordered following the arrangement in Figs A1 and A2: the first 16 elements correspond to the measurements in the first row of those figures, the next 12 elements correspond to the second row, etc. The key takeaway from this figure is that there is little covariance between clusters in different redshift bins, but that there is significant covariance between different richness bins within the same redshift slice.

\subsection{Correction for observational systematics}

If the density of galaxy clusters is modulated by observing conditions, the variation in observing conditions across the survey will induce artificial power in the clustering of clusters. To correct for this potential source of systematic error, we evaluate several measures of observation quality at each cluster's location - including sky flux, dust extinction, size of the point spread function (PSF) and survey depth - and then bin the clusters by these measures. We 


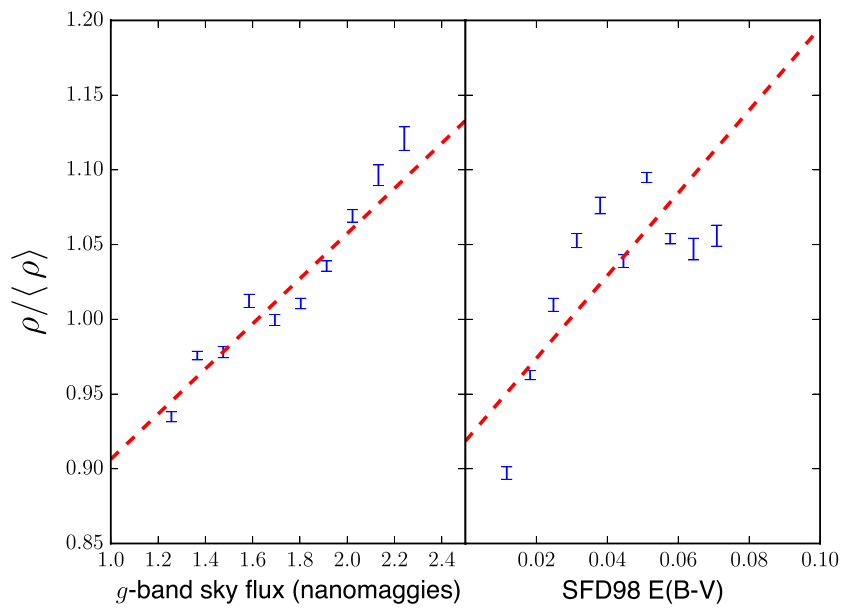

Figure 2. The measured variation in redMaPPer cluster density with $g$-band sky flux and $E(B-V)$ dust extinction measured by Schlegel et al. (1998). Dashed lines show the linear fits that we use to correct our clustering measurements for these variations.

then determine the area sampled by each bin, and calculate the corresponding cluster number density. In the absence of observational systematics, we expect the cluster density to be independent of the various measures of observation quality.

Fig. 2 shows how the redMaPPer cluster density varies with both sky flux in the $g$ band (left-hand panel) and $E(B-V)$ dust extinction measured by Schlegel, Finkbeiner \& Davis (1998, right-hand panel). We have selected these two potential sources of systematic contamination because they exhibit the strongest effect on the redMaPPer cluster density. Variations in $g$-band sky flux and dust extinction can lead to $\sim 10$ per cent changes in the cluster density. In order to correct our analysis for these effects, we introduce a systematic weight, $w_{i}^{\text {sys }}$, for the $i$ th cluster:

$w_{i}^{\text {sys }}=\frac{A+B s_{i}}{\bar{n}}$,

where $\bar{n}$ is the average cluster density, $s_{i}$ is the measure of the observational systematic evaluated at the $i$ th cluster and $A+B s_{i}$ is the linear fit to the observed variations in cluster density shown as the dashed lines in Fig. 2. The weight $w_{i}^{\text {sys }}$ is the relative oversampling of regions that have observation quality described by $s_{i}$. Consequently, we can correct for this systematic by applying $w_{i}^{\text {sys }}$ to each random point. We choose to only weight the random catalogue by sky flux in the $g$ band as the correlation between this systematic and the redMaPPer cluster density appears particularly tight. In Section 7.5, we determine the level of systematic uncertainty introduced into our parameter constraints by this choice.

\section{ANALYSIS}

Given bias parameters $b^{\alpha}$ for each richness/redshift bin and a cosmological model, we can compute the predicted $w^{\alpha \beta}(\theta)$ using equations (3) and (4). We compute the matter power spectrum, $P(k, z)$, in equation (4) using $\mathrm{CAMB}^{3}$ (Lewis, Challinor \& Lasenby 2000) with the Halofit prescription for the non-linear power spectrum (Smith et al. 2003) including the updates of Takahashi et al. (2012). At scales larger than $\sim 5 \mathrm{Mpc}$, the influence of baryons on the matter power spectrum is expected to be at most a few per cent (e.g. van

\footnotetext{
${ }^{3}$ http://camb.info
}

Daalen et al. 2011). Since our analysis only uses scales larger than $5 h^{-1} \mathrm{Mpc}$, the effects of baryons on the matter power spectrum are well below the statistical uncertainty of our measurements. The accuracy of Halofit should therefore be sufficient for this analysis.

\subsection{Bias-only model}

One way to model the bias, $b^{\alpha}$, of each bin of clusters is to simply treat the $b^{\alpha}$ as free parameters. The results of fitting such a model to the observed cluster correlation functions are presented in Section 6. While constraints on the $b^{\alpha}$ are potentially useful for a cosmological analysis of redMaPPer clusters, they suffer from the drawback that the $b^{\alpha}$ depend on the richness and redshift bin choices.

An alternate and bin-independent approach is to parametrize the relationship between cluster bias and the observed richness and redshift of each cluster. This parametrization can then be constrained by fitting to the measured angular correlation function. We adopt a simple model for the richness and redshift dependence of the bias:

$b(\lambda, z)=A_{\lambda}\left(\frac{\lambda}{\lambda_{0}}\right)^{\alpha_{\lambda}}\left(\frac{1+z}{1+z_{0}}\right)^{\beta_{\lambda}}$

where $A_{\lambda}, \alpha_{\lambda}$ and $\beta_{\lambda}$ are free parameters. We set $\lambda_{0}=35$ and $z_{0}=0.25$ throughout; these values are chosen because they are roughly the median richness and redshift for our sample, respectively.

When constraining the bias-richness parametrization and the mass-richness relation of redMaPPer clusters, we restrict the application of these parametrizations to clusters with $\lambda \geq 20$. As discussed in Rykoff et al. (2014), our a priori expectation is that the connection between galaxy clusters and individual dark matter haloes is very clean at high richness, but may become more problematic with decreasing richness. The richness threshold $\lambda \geq 20$ is one we believe to be very conservative. However, the lowest richness clusters with richness $5<\lambda<20$ are still expected to be perfectly good mass tracers. Consequently, we model the bias of the richness bin $5<\lambda<20$ with a single bias parameter, $b^{0}$ (with each redshift bin having a different $b^{0}$ parameter). Because the cross-correlations between the $5<\lambda<20$ clusters and the $\lambda \geq 20$ clusters contain information about the biases of the $\lambda \geq 20$ clusters, and because there are many clusters with $5<\lambda<20$, including these crosscorrelations in our analysis significantly reduces the statistical error of our bias measurements.

For the $\lambda \geq 20$ clusters, equation (10) yields the bias for a cluster at a particular richness and redshift. What we measure, however, is the bias averaged over a richness/redshift bin. To model the bias of a richness/redshift bin, we simply average equation (10) over the bin:

$b^{\alpha}=\frac{1}{N_{\alpha}} \sum_{i}^{N_{\alpha}} b\left(\lambda_{i}, z_{i}\right)$,

where the sum runs over the $N_{\alpha}$ clusters in the richness/redshift bin $\alpha$. Our model therefore takes into account the richness and redshift distribution of all of the clusters in the bin. Note that at this stage, our modelling is completely independent of the mass-richness relation.

\subsection{Mass-observable parametrization}

Above, we parametrized the bias of the redMaPPer clusters in terms of the cluster richness and redshift. Alternatively, if both the massbias relationship and the mass-richness relation for redMaPPer clusters is known, one can use these relations to predict the clustering 
bias - and therefore the angular correlation function - of the cluster population. Parametrizing in this way allows one to use the data to constrain the mass-richness model for the redMaPPer clusters.

For clusters with $\lambda \geq 20$, we model the bias as a function of richness and redshift with

$b(\lambda, z)=\int \mathrm{d} \ln M b(\ln M, z) P(\ln M \mid \lambda, z)$,

where $P(\ln M \mid \lambda, z)$ describes the probability of a cluster having $\log$-mass $\ln M$ given that it has observed richness $\lambda$ and redshift $z$. The term $b(\ln M, z)$ is the bias of a cluster with $\log$-mass $\ln M$ and redshift $z$. We adopt the best-fitting model for $b(\ln M, z)$ from Tinker et al. (2010), where $b(\ln M, z)$ was calibrated using $N$-body simulations.

We consider a Gaussian model for $P(\ln M \mid \lambda, z)$ :

$P(\ln M \mid \lambda, z)=\frac{1}{\sqrt{2 \pi \sigma_{\ln M}^{2}}} \exp \left[-\frac{(\ln M-\langle\ln M \mid \lambda, z\rangle)^{2}}{2 \sigma_{\ln M}^{2}}\right]$.

To parametrize $\langle M \mid \lambda, z\rangle$, we adopt the model

$\langle M \mid \lambda, z\rangle=A\left(\lambda / \lambda_{0}\right)^{\alpha}\left(\frac{1+z}{1+z_{0}}\right)^{\beta}$.

We treat $\sigma_{\ln M}, \ln A, \alpha$ and $\beta$ as free parameters. This parametrization is equivalent to

$\langle\ln M \mid \lambda\rangle=\ln A+\alpha \ln \left(\lambda / \lambda_{0}\right)+\beta \ln \left(\frac{1+z}{1+z_{0}}\right)-\frac{1}{2} \sigma_{\ln M}^{2}$,

which can be directly substituted into equation (13). ${ }^{4}$ We set $\lambda_{0}=35.0$ and $z_{0}=0.25$ as before.

\subsubsection{Line-of-sight cluster projections}

Some redMaPPer objects may in fact be projections of multiple systems along the line of sight. We consider only projections of two haloes since projections of three or more haloes are significantly less likely and will therefore have a subdominant impact on our results. Since projections typically occur between clusters that are separated by $\Delta z \lesssim 0.02$, and the width of our redshift bins is $\Delta z \sim 0.07$, we ignore redshift differences between the two haloes. In this section, we suppress the $z$ dependence of the bias for notational convenience. We also note that in addition to projections, a small fraction (roughly 1 to 2 per cent) of redMaPPer-detected objects are expected to be affected by photometric failures in the SDSS pipeline. We ignore this effect here, however, as it contributes a level of bias to our measurements that is well below our statistical uncertainty.

We model the bias of clusters in the presence of projections via

$b(\lambda)=(1-f) b^{\text {no-proj }}(\lambda)+f b^{\text {proj }}(\lambda)$,

where $b^{\text {no-proj }}(\lambda)$ is given by equation (12), and $b^{\text {proj }}(\lambda)$ is the bias of projected clusters of total observed richness $\lambda$. The parameter $f$ can be thought of as the fraction of redMaPPer objects that are projected systems (we discuss constraints on $f$ in more detail at the end of this section). To complete our model, we need to determine an expression for $b^{\text {proj }}(\lambda)$.

Consider a projected cluster with richness $\lambda$ that is a blend of two haloes of mass $M_{1}$ and $M_{2}$. We assume that the total richness of the

\footnotetext{
${ }^{4} \mathrm{We}$ have also considered the alternate (but similar) parametrization $\langle\ln M \mid \lambda\rangle=\ln A+\alpha \ln \left(\lambda / \lambda_{0}\right)+\beta \ln \left(\frac{1+z}{1+z_{0}}\right)$ but find that this parametrization is more sensitive to the value of $\sigma_{\ln M}$
}

projected system is equal to the sum of the richnesses of the two projected haloes: $\lambda=\lambda_{1}+\lambda_{2}$, where $\lambda_{1}$ and $\lambda_{2}$ are the richnesses of the haloes of mass $M_{1}$ and $M_{2}$, respectively. We define $q=\lambda_{1} / \lambda$, so that $q \leq 1.0$. Without loss of generality, we assume that $\lambda_{1}>\lambda_{2}$ and so $q \geq 0.5$.

The bias of the projected pair of haloes, $b^{\text {proj }}(\lambda)$, should be higher than the bias of either of the individual haloes since projections occur preferentially in high-density regions. Consequently, $b^{\text {proj }}(\lambda)$ $\geq b\left(\lambda_{1}\right)$, and thus

$b^{\text {proj }}(\lambda)>\int \mathrm{d} M_{1} b\left(M_{1}\right) P\left(M_{1} \mid q \lambda\right)$.

Furthermore, $b^{\text {proj }}$ should increase as the separation between $M_{1}$ and $M_{2}$ decreases. In the limit of zero separation, one would expect the effective bias to be $b\left(M_{1}+M_{2}\right)$, and hence $b^{\text {proj }}(\lambda) \leq b\left(M_{1}+M_{2}\right)$. Since our model has $M \propto \lambda^{\alpha}$, then

$M_{2} \sim M_{1}\left(\frac{1-q}{q}\right)^{\alpha}$.

We therefore obtain an upper limit to the bias of the projected system

$b^{\text {proj }}(\lambda)<\int \mathrm{d} M_{1} b\left(M_{1}\left[1+\left(\frac{1-q}{q}\right)^{\alpha}\right]\right) P\left(M_{1} \mid q \lambda\right)$.

We can smoothly interpolate between the lower limit in equation (17) and the upper limit in equation (19) by introducing a new parameter, $g \in[0,1]$, that scales the $((1-q) / q)^{\alpha}$ term. Our model for the bias including projection effects is then

$$
\begin{aligned}
b(\lambda)= & (1-f) \int \mathrm{d} M b(M) P(M \mid \lambda) \\
& +f \int \mathrm{d} M_{1} b\left(M_{1}\left[1+g\left(\frac{1-q}{q}\right)^{\alpha}\right]\right) P\left(M_{1} \mid q \lambda\right),
\end{aligned}
$$

where $q \in[0.5,1]$ and $g \in[0,1]$. In the limit that $q=1$, there is no mass or richness in the less rich halo and equation (20) recovers the unprojected expression (equation 12) as expected.

We adopt flat priors on $q$ and $g$ across their allowed ranges. Note that describing the effects of projections using a single $q$ and a single $g$ for all clusters is somewhat unrealistic since the values of these parameters are likely different for different projected systems. However, by keeping $q$ and $g$ constant across all clusters, we are effectively extremizing the effects of projections so this is a conservative approach.

As for the fraction of clusters that are projections, $f$, we adopt the prior $f=0.1 \pm 0.04$ utilized in S16. The S16 estimate of $f$ is derived using two different approaches. The first approach is based on the Rykoff et al. (2014) estimate of the projection fraction. Rykoff et al. (2014) estimated a projection fraction of 5 per cent for highrichness clusters by performing cluster finding on mock clusters inserted randomly into SDSS imaging. S16 scale this estimate by a factor of $\eta=1.8 \pm 0.4$ to account for the fact that redMaPPer clusters are clustered on the sky, and thus more likely to be projections than the randomly located mock clusters used in Rykoff et al. (2014). This scaling results in $f=9$ percent \pm 2 percent. The second approach relies on Rozo et al. (2015b), which found that approximately 6 per cent \pm 2 per cent of the cluster richness is due to projected galaxies. S16 argue that this value translates into $f=0.12 \pm 0.04$ after making some reasonable assumptions about the amount of richness that a projection contributes to a cluster. Finally, S16 combine these two estimates to arrive at $f=0.1 \pm$ 0.04 , the range adopted in this work. 


\subsection{Assembly bias}

It is commonly assumed that the clustering amplitude of dark matter haloes depends only on the halo mass. Assembly bias refers to the dependence of halo clustering on additional properties of the haloes, such as assembly history or concentration. Assembly bias has been observed in simulations (e.g. Gao et al. 2005; Wechsler et al. 2006; Jing et al. 2007) and motivated by theory (e.g. Dalal et al. 2008). Recently, using a sample of SDSS-identified redMaPPer clusters very similar to that used in this work, Miyatake et al. (2016, hereafter M16) have measured the dependence of cluster clustering on $\bar{R}_{\text {mem }}$, defined as the mean separation of cluster member galaxies from the cluster centre. If this dependence of clustering on $\bar{R}_{\text {mem }}$ results from a form of assembly bias, it will complicate the simple model for the bias of a bin of redMaPPer clusters that we have constructed above.

If unaccounted for, assembly bias is a potential source of systematic error in our analysis. The $b(M)$ from Tinker et al. (2010) is calibrated by averaging over all haloes of fixed mass $M$ in simulations. The redMaPPer catalogue, on the other hand, is generated by selecting on richness, $\lambda$. Let $\gamma$ represent some additional property of haloes besides mass that affects their clustering, such as assembly history or concentration. If the distribution of $\gamma$ for a richnessselected sample differs from that of a mass-selected sample, then the clustering amplitude of the redMaPPer clusters may differ from that predicted by $b(M)$, resulting in biased parameter constraints. This effect has been estimated to be negligible for SDSS-identified clusters by Wu, Rozo \& Wechsler (2008). However, recent measurements by M16 suggest that assembly bias effects may be non-negligible for our redMaPPer cluster sample. Consequently, we explore the impact of assembly bias on our mass-richness calibration results in more detail below. Although we focus on the dependence of halo clustering on $\bar{R}_{\text {mem }}$, our analysis is quite general and could easily be adapted to any quantity relevant to assembly bias effects. Finally, we note that in addition to dependence of halo bias on assembly history or concentration, the mass-richness relation of redMaPPer clusters may also be dependent on assembly history or halo concentration (Zentner et al. 2005; Mao, Williamson \& Wechsler 2015).

M16 consider the dependence of cluster clustering on the mean distance between the cluster centre and the cluster member galaxies, $\bar{R}_{\text {mem }}$ :

$\bar{R}_{\mathrm{mem}}=\sum_{i} R_{i} P_{i}$

where $R_{i}$ is the physical separation between the $i$ th member galaxy and the corresponding halo centre, and $P_{i}$ is the membership probability of the $i$ th galaxy as determined by the redMaPPer algorithm. M16 divide their cluster sample into two sets with roughly identical richness and redshift distributions, but with different $\bar{R}_{\text {mem }}$ distributions. For both of these cluster samples, M16 measure the weak lensing signals around the clusters and fit these measurements with a halo model. They find that the one-halo terms for both cluster samples are consistent within errors (suggesting that the two samples have similar halo-mass distributions), but that the two-halo clustering amplitudes are different. M16 also measure halo clustering directly, and find that the ratio of the clustering amplitudes for the two samples is consistent with that found in their weak lensing analysis. These results suggest that the clustering of redMaPPer clusters depends not only on the halo masses of these clusters, but also on $\bar{R}_{\text {mem }}$ (see also More et al. 2016, for further discussion).

Rather than working with $\bar{R}_{\text {mem }}$ directly, it is easiest to consider $\bar{R}_{\text {mem }}$ normalized by the mean value of $\bar{R}_{\text {mem }}$ at a given richness and redshift. We define

$\Delta \equiv \frac{\bar{R}_{\text {mem }}-\left\langle\bar{R}_{\text {mem }} \mid \lambda, z\right\rangle}{\left\langle\bar{R}_{\text {mem }} \mid \lambda, z\right\rangle}$,

where $\left\langle\bar{R}_{\text {mem }} \mid \lambda, z\right\rangle$ is the mean $\bar{R}_{\text {mem }}$ for clusters of richness $\lambda$ and redshift $z$. We approximate $\left\langle\bar{R}_{\text {mem }} \mid \lambda, z\right\rangle$ by fitting a spline to the average value of $\bar{R}_{\text {mem }}$ computed across 10 bins of richness and 5 bins of redshift.

The dependence of halo clustering on $\Delta$ can in principle affect our analysis in two ways. First, the mass-richness relationship, $P(M \mid \lambda)$, can depend on $\Delta$ (see e.g. Mao et al. 2015). Since the clustering amplitude depends on the halo mass, any dependence of the mass-richness relationship on $\Delta$ would induce variation in the clustering amplitude with $\Delta$. Secondly, the relationship between halo mass and clustering, $b(M)$, could directly depend on $\Delta$. In principle, both of these effects could be relevant to the redMaPPer clusters. However, M16 have found that their two cluster samples have nearly identical richness distributions (by construction), very different $\Delta$ distributions (also by construction), but very nearly identical lensing signals in the one-halo regime, i.e. nearly identical mean halo masses. Their results suggest (but do not require) that $\Delta$ does not severely impact the mass-richness relation, but does significantly affect the clustering amplitude. That is, we should focus on a model, $b(M, \Delta)$, in which the halo clustering amplitude is directly modulated by $\Delta$. Again, we emphasize that although we have defined $\Delta$ in terms of $\bar{R}_{\text {mem }}$, the model presented below would work equally well for any quantity relevant to assembly bias, such as halo concentration.

We adopt a linear model for the dependence of bias on $\Delta$ :

$b(M, z, \Delta)=b(M, z)\left(c_{0}+c_{1} \Delta\right)$,

where $b(M, z)$ is the bias model from Tinker et al. (2010). Given this dependence of the bias on $\Delta$, equation (12) must be replaced with

$$
\begin{aligned}
b(\lambda, z, \Delta) & =\int \mathrm{d} M P(M \mid \lambda, z, \Delta) b(M, z, \Delta) \\
& =\left(c_{0}+c_{1} \Delta\right) \int \mathrm{d} M P(M \mid \lambda, z) b(M, z) .
\end{aligned}
$$

In going from the first to the second line, we have assumed that the mass-richness relationship is independent of $\Delta$. We note that M16 found consistent values for the average mass of clusters in bins of $\Delta$, but this does not require that there be no dependence of the mass-richness relationship on $\Delta$. The bias for a richness/redshift bin can then be modelled as in equation (11), except now we include the dependence of the bias on $\Delta$ :

$b^{\alpha}=\frac{1}{N_{\alpha}} \sum_{i}^{N_{\alpha}} b\left(\lambda_{i}, z_{i}, \Delta_{i}\right)$.

While our model has introduced two new parameters $-c_{0}$ and $c_{1}$ - in practice these two are related. Specifically, by construction, $b(M)$ is the halo bias averaged over all haloes of a given mass. Consequently, one must obtain the Tinker et al. (2010) mass-bias relation when averaging over all haloes of a given mass:

$b(M, z)=\langle b\rangle_{M, z} \equiv \int \mathrm{d} \Delta b(M, z, \Delta) P(\Delta \mid M, z)$,

where we have defined the quantity $\langle b\rangle_{M, z}$, which represents the bias averaged over the $\Delta$ distribution, $P(\Delta \mid M, z)$, at fixed mass and redshift. Satisfying the above equation and substituting the ansatz 
of equation (23) yields the constraint

$c_{0}+c_{1}\langle\Delta\rangle_{M, z}=1$,

where we have defined

$\langle\Delta\rangle_{M, z} \equiv \int \mathrm{d} \Delta \Delta P(\Delta \mid M, z)$

as the mean $\Delta$ at fixed $M$ and $z$.

Similarly, we can compute the average bias over the $\Delta$ distribution at fixed richness and redshift:

$$
\begin{aligned}
\langle b\rangle_{\lambda, z} & \equiv \int \mathrm{d} \Delta b(\lambda, z, \Delta) P(\Delta \mid \lambda, z) \\
& =\int \mathrm{d} \Delta\left(c_{0}+c_{1} \Delta\right) P(\Delta \mid \lambda, z) \int \mathrm{d} M P(M \mid \lambda, z) b(M, z) \\
& =\left(c_{0}+c_{1}\langle\Delta\rangle_{\lambda, z}\right) b_{0}(\lambda, z) \\
& =\left[1+c_{1}\left(\langle\Delta\rangle_{\lambda, z}-\langle\Delta\rangle_{M, z}\right)\right] b_{0}(\lambda, z) .
\end{aligned}
$$

Here, we have defined $b_{0}(\lambda, z)$ via equation (12), i.e. $b_{0}(\lambda, z)$ is the bias we predict in the absence of assembly bias. We have further defined

$\langle\Delta\rangle_{\lambda, z} \equiv \int \mathrm{d} \Delta \Delta P(\Delta \mid \lambda, z)$

in analogy with equation (28).

Notice that from equation (29) it is clear that if the $\Delta$ distribution for a richness-selected sample of clusters is identical to the $\Delta$ distribution of haloes at fixed mass, then $\langle\Delta\rangle_{\lambda, z}=\langle\Delta\rangle_{M, z}$ and the perturbation term to our prediction exactly cancels out, as it should. We can, however, simplify our expression just a bit more by noting that by definition, $\langle\Delta\rangle_{\lambda, z}=0$. Consequently,

$\langle b\rangle_{\lambda, z}=\left[1-c_{1}\langle\Delta\rangle_{M, z}\right] b_{0}(\lambda, z)$.

Thus, we see that the quantity $\langle\Delta\rangle_{M, z}$ governs the effects of assembly bias on our measurements.

To complete our model for assembly bias, we must determine the values of $c_{1}$ and $\langle\Delta\rangle_{M, z}$. In the absence of a true mass-selected cluster sample, we rely instead on a cluster sample selected on Sunyaev-Zel'dovich (SZ) decrement to estimate $\langle\Delta\rangle_{M, z}$. An SZselected sample makes a reasonable proxy for mass selection because the scatter in the SZ-mass relation is low (e.g. Angulo et al. 2012). One could also imagine using weak lensing masses from e.g. von der Linden et al. (2014) to construct an approximately mass-selected sample. However, the small number of clusters with individually measured weak lensing masses means that the sample size would be small and the statistical noise would be large. Even with many SZ-selected clusters, the statistical errors on the measured $\langle\Delta\rangle_{M, z}$ are quite large.

Our SZ-selected cluster sample is derived from the union catalogue of SZ-detected clusters from (Planck Collaboration XXVII 2015 c), matched to redMaPPer clusters in order to calculate $\lambda$ and $\Delta$. Note that since all Planck clusters in the redshift range of our analysis and in the SDSS footprint are identified by redMaPPer, this results in a complete sample of SZ-selected clusters (Rozo et al. 2015a).

The $\Delta$ distributions of the redMaPPer and Planck-selected clusters are compared in Fig. 3. The top panel of this figure shows the distribution of clusters in richness and $\Delta$, while the bottom panel shows the distributions as a function of $\Delta$ alone. The widths of the curves in the bottom panel are the Poisson errors for each $\Delta$ bin. Making the simplifying assumption that $\langle\Delta\rangle_{M, z}$ is not strongly dependent on mass or redshift, $\langle\Delta\rangle_{M, z}$ is just the average value of
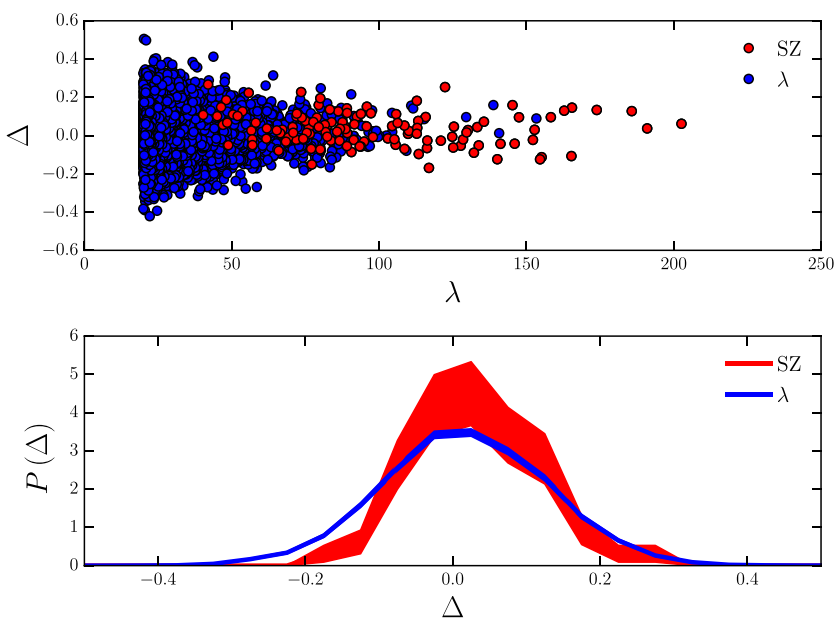

Figure 3. Illustration of how $\mathrm{SZ}$ selection affects the distribution of cluster member concentrations. The top panel shows the distribution of clusters in richness $(\lambda)$ and cluster member concentration $(\Delta)$ for a richness-selected sample (blue points) and an SZ-selected sample from Planck Collaboration XXVII (2015c) (red points). Values of $\lambda$ and $\Delta$ for the SZ-selected clusters are computed by matching to the redMaPPer catalogue. The bottom panel shows the $\Delta$ distributions for these two samples, with the widths of the curves indicating the Poisson error bars. Comparing the red and blue curves in the bottom panel suggests that richness-selected clusters tend to have slightly lower values of $\Delta$ than SZ-selected clusters, meaning that richnessselected clusters are more compact on average. As described in the text, the difference in the $\Delta$ distributions for the two samples can be used to compute the effects of assembly bias on our analysis.

$\Delta$ for all of the clusters in the SZ-matched sample. Performing this average, we find $\langle\Delta\rangle_{M, z}=0.032 \pm 0.007$. The fact that $\langle\Delta\rangle_{M, z}>0$ (measured from the SZ-selected sample) means that SZ-selected clusters on average have larger values of $\bar{R}_{\text {mem }}$ than redMaPPer clusters, i.e. redMaPPer clusters are preferentially more compact than SZ-selected clusters.

We now turn to estimating $c_{1}$, the remaining parameter in our model. Measuring $c_{1}$ with the SZ-selected sample is difficult because the small number of SZ-selected clusters means that any correlation function measurement with these clusters is very noisy. Instead, we estimate $c_{1}$ using the redMaPPer sample in the following way. In analogy with equation (23), we define

$b(\lambda, z, \Delta)=\langle b\rangle_{\lambda, z}\left(d_{0}+d_{1} \Delta\right)$.

Since $\langle\Delta\rangle_{\lambda, z}=0$ by definition, we must have $d_{0}=1$. By measuring the correlation function in bins of $\Delta$, we can fit for $d_{1}$. We then adopt the approximation $c_{1} \approx d_{1}$.

For the purposes of estimating $d_{1}$, we divide the cluster sample into three bins of $\Delta$ and measure the angular clustering of the clusters in these bins. We then fit these measurements treating the bias in each $\Delta$ bin as a free parameter. The constraints on the bias parameters as a function of $\Delta$ are shown as the data points with error bars in Fig. 4. The position on the $x$-axis of the data points is the average value of $\Delta$ for that bin. We model the bias measurements shown in Fig. 4 with

$b_{\gamma}=\sum_{i}^{N_{\gamma}} b\left(\lambda_{i}, z_{i}\right)\left(1+d_{1} \Delta_{i}\right)$,

where the sum runs over all $N_{\gamma}$ clusters in the $\gamma$ th $\Delta$ bin and $d_{1}$ is treated as a free parameter. For $b\left(\lambda_{i}, z_{i}\right)$, we use the best-fitting $b(\lambda$, $z$ ) relation from the bias-only analysis described in Section 5.1. 


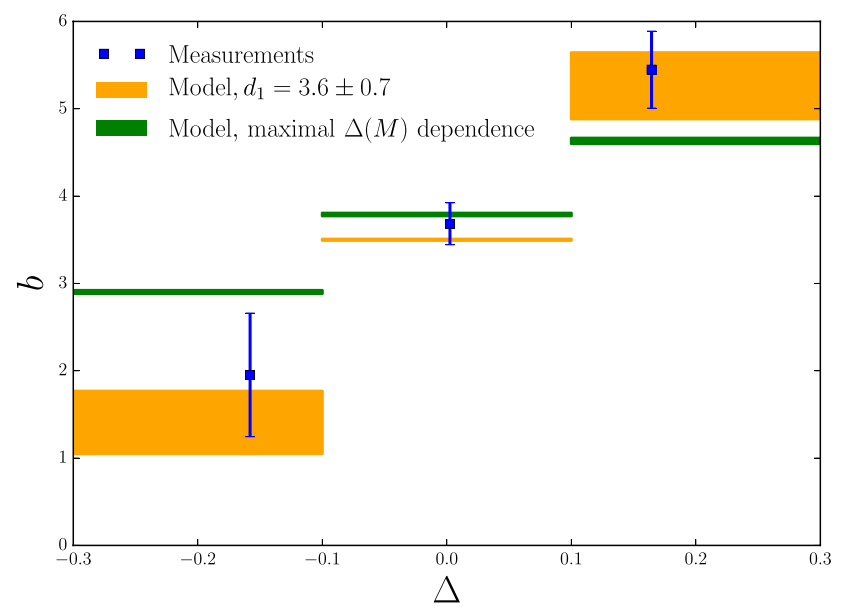

Figure 4. Clustering bias $(b)$ as a function of cluster member concentration $(\Delta)$ for three $\Delta$ bins (blue points). The cluster sample used in this plot has been restricted to $\lambda \geq 20$. Orange regions show the best-fitting model prediction of equation (23) for the mean bias of each $\Delta$ bin; this model assumes that the clustering of redMaPPer clusters depends directly on $\Delta$. The vertical extent of the regions indicates the range allowed by the uncertainty on the model parameter $d_{1}$. The green regions represent the biases predicted by a model (equation 34) that instead assumes that redMaPPer clustering depends on $\Delta$ because cluster mass and $\Delta$ are maximally correlated. The vertical extent of the green regions represents the error on the mean bias in this model. The relatively poor fit of equation (34) to the clustering bias measurements suggests that dependence of $\Delta$ on cluster mass is not sufficient to explain the observed dependence of redMaPPer clustering on $\Delta$.

The fitting procedure described above yields a constraint of $d_{1}=3.6 \pm 0.7$. We therefore find statistically significant evidence $(5 \sigma)$ that the clustering of redMaPPer clusters increases with $\Delta$, in agreement with the results of M16. Using this constraint on $d_{1}$, we can compute a model prediction (using equation 33) for the bias of every redMaPPer cluster. These predictions, averaged over all clusters in each $\Delta$ bin, are shown as the solid orange bars in Fig. 4. The extent of these bars along the $x$-axis indicates the width of the $\Delta$ bin, while the extent along the $y$-axis indicates the range allowed by the uncertainty on $d_{1}$. It is clear from the figure that our simple linear model for the dependence of the bias on $\Delta$ (equation 33) provides a reasonable fit to the measured biases. We have performed similar fits after dividing the redMaPPer clusters into richness bins, and find no significant evidence that $d_{1}$ varies with richness.

We now have all the ingredients necessary to compute $c_{0}$ and $c_{1}$. We approximate $c_{1} \approx d_{1}$ and set $c_{0}=1-c_{1}\langle\Delta\rangle_{M, z}$. The bias for a richness/redshift bin can then be computed using equation (25). In computing $c_{0}$ and $c_{1}$, we have made two significant assumptions: first, that the SZ-selected sample could be used as a reasonable proxy for a mass-selected sample when computing $\langle\Delta\rangle_{M, z}$, and second, that we could approximate $c_{1} \approx d_{1}$. To account for the systematic error introduced by these assumptions, we take the approach of allowing $\langle\Delta\rangle_{M, z}$ and $c_{1}$ to vary in our analysis across wide, flat priors. The central values of these priors are chosen to be $\langle\Delta\rangle_{M, z}=0.032$ and $c_{1}=3.6$, i.e. the values measured above. For the widths of the priors, we allow $\langle\Delta\rangle_{M, z}$ and $c_{1}$ to vary between 50 per cent and 150 per cent of their central values. These priors are summarized in Table 1.

Since we find $\langle\Delta\rangle_{M, z}>0$, redMaPPer clusters are slightly more concentrated than SZ-selected clusters and therefore have lower value of $\Delta$ on average. Since we find $c_{1}>0$, lower values of $\Delta$ correspond to lower clustering biases. Consequently, for fixed measurements of $w^{\alpha \beta}(\theta)$, a model that includes a form of assembly bias due to $\bar{R}_{\text {mem }}$ is expected to yield a somewhat higher normalization of the mass-richness relation. Indeed, as we show in Section 7.3, this is what we find.

\subsubsection{Could the dependence of redMaPPer clustering on $\Delta$ be due to dependence of $\Delta$ on cluster mass?}

Given the measured dependence of the bias with $\Delta$ shown in Fig. 4 , an interesting question to ask is whether or not these measurements could be explained by a dependence of $\Delta$ on cluster mass. If higher mass clusters tended to have higher values of $\Delta$, these clusters would also have higher biases because $b(M)$ is an increasing function. If this were the case, the measured dependence of the bias on $\Delta$ would not be a form of assembly bias. ${ }^{5}$

The dependence of cluster mass on $\Delta$ would have to be fairly steep in order to explain the observed dependence of the bias on $\Delta$. However, if the dependence of mass on $\Delta$ were too steep, the resultant scatter in the redMaPPer mass-richness relation would be larger than the observed scatter. The most extreme model is that all of the scatter in the mass-richness relation is due to the dependence of mass on $\Delta$. If we can show that even this extreme model cannot explain the observed dependence of bias on $\Delta$, then this constitutes evidence that the dependence of redMaPPer clustering on $\Delta$ is not due to dependence of $\Delta$ on halo mass. Note that if all of the scatter in the mass-richness relation were due to $\Delta$, one could calibrate a new mass-richness- $\Delta$ relationship with zero scatter.

To test the extreme model described above, we assign masses to the redMaPPer clusters assuming that scatter in the mass-richness relation is maximally correlated with scatter in $\Delta$. The $i$ th cluster is assigned a mass using

$\ln M_{i}=\left\langle\ln M \mid \lambda_{i}, z_{i}\right\rangle+\frac{\Delta_{i}}{\sigma_{\Delta}\left(\lambda_{i}\right)} \sigma_{\ln M}\left(\lambda_{i}\right)$,

where we use our best-fitting model for $\left\langle\ln M \mid \lambda_{i}, z_{i}\right\rangle$ and $\sigma_{\Delta}(\lambda)$ is the standard deviation of $\Delta$ as a function of cluster richness. We compute $\sigma_{\Delta}(\lambda)$ by binning clusters in richness and computing $\sigma_{\Delta}$ in each richness bin; $\sigma_{\Delta}(\lambda)$ is computed at arbitrary richness by linear interpolation. We assume that the scatter in the mass-richness relation is described by

$\sigma_{\ln M}^{2}(\lambda)=\alpha^{2}\left[\sigma_{\ln \lambda \mid M}^{2}+(1 / \lambda)\right]$.

The form of this scatter reflects the population statistics of dark matter substructures in host haloes as found in numerical simulations (e.g. Boylan-Kolchin et al. 2010; Mao et al. 2015). We assume that the intrinsic scatter in mass at fixed richness for high-mass clusters (where the $1 / \lambda$ term becomes negligible) is $\sigma_{\ln M \mid \lambda}=\alpha \sigma_{\ln \lambda \mid M}=0.3$. This is a conservative choice; Rozo \& Rykoff (2014) and Rozo et al. (2015a) constrain $\sigma_{\ln M \mid \lambda} \approx 0.25$, including the Poisson contribution. By setting $\sigma_{\ln M \mid \lambda}=0.3$, we are overestimating the impact that mass scatter can have on the clustering bias prediction.

The mass assignments described by equation (34) effectively attribute all of the scatter in the mass-richness relation to variation in $\Delta$. With the masses assigned to each cluster using the model of

\footnotetext{
${ }^{5}$ The measurements of M16 suggest that $\Delta$ does not depend strongly on cluster mass since the one-halo contributions to the weak lensing signals of these two samples are nearly identical. Our goal here, however, is to determine whether or not our clustering measurements support the assembly bias picture in the absence of the mass measurements by M16.
} 
Table 1. Priors and posteriors on parameters of mass-richness parametrization. The parameters $\ln A, \alpha, \beta$ and $\sigma$ govern the amplitude, slope in richness, slope in mass and scatter of the mass-richness relationship of redMaPPer clusters, respectively. The parameter $f$ is the fraction of clusters that are projections, $q$ is the fraction of mass in the dominant halo of a projection and $g$ governs the magnitude of projection effects. The parameter $b_{\text {scaling }}$ controls the uncertainty in the model $b(M)$ from Tinker et al. (2010). The parameters $\langle\Delta\rangle_{M, z}$ and $c_{1}$ govern the assembly bias of redMaPPer clusters. The parameters $f, q, g, b_{\text {scaling }},\langle\Delta\rangle_{M, z}$, $c_{1}$ and $\sigma$ are all nuisance parameters that we marginalize over. Priors of the form $[A, B]$ are flat with minimum and maximum given by $A$ and $B$; priors of the form $A \pm B$ are Gaussian with mean given by $A$ and standard deviation given by $B$. Posteriors are represented in terms of the mean and standard deviation.

\begin{tabular}{lccc}
\hline Parameter & Description & Prior & Posterior \\
\hline$b^{0, z_{0}}$ & Bias of $5<\lambda<20,0.18<z<0.26$ clusters & {$[0.2,10.0]$} & $1.85 \pm 0.05$ \\
$b^{0, z_{1}}$ & Bias of $5<\lambda<20,0.26<z<0.33$ clusters & {$[0.2,10.0]$} & $1.74 \pm 0.05$ \\
$\ln \left(A / \mathrm{M}_{\odot}\right)$ & Amplitude of mass-richness relation & {$[30.0,35.0]$} & $33.66 \pm 0.18$ \\
$\alpha$ & Richness scaling of mass-richness relation & {$[-0.5,4.0]$} & $1.18 \pm 0.16$ \\
$\beta$ & Redshift scaling of mass-richness relation & {$[-20.0,20.0]$} & $1.86 \pm 2.4$ \\
$\sigma$ & Scatter in mass-richness relation & {$[0.05,0.5]$} & - \\
$f$ & Projection fraction & $0.10 \pm 0.04$ & - \\
$q$ & Projection effects & {$[0.5,1.0]$} & - \\
$g$ & Projection effects & {$[0.0,1.0]$} & - \\
$b_{\text {scaling }}$ & Uncertainty in $b(M)$ & $0.00 \pm 0.06$ & - \\
$\langle\Delta\rangle_{M, z}$ & Assembly bias & {$[0.011,0.053]$} & - \\
$c_{1}$ & Assembly bias & {$[0.95,3.95]$} & \\
\hline
\end{tabular}

equation (34), we compute the average bias of each $\Delta$ bin in Fig. 4 using the $b(M)$ relation from Tinker et al. (2010). These average biases and the corresponding error bars are shown as the green solid regions in Fig. 4. It is clear from the figure that the no-assemblybias model is a worse fit to the bias measurements than our fiducial assembly bias model (i.e. equation 23). We find $\chi^{2}=5.4$ for the model of equation (34) with one degree of freedom (since we fit for the mean bias), corresponding to a probability to exceed of 2 per cent. The measured bias values can therefore be taken as weak evidence that the observed dependence of redMaPPer clustering on $\Delta$ is not due to dependence of $\Delta$ on cluster mass. This statement is conservative since we have assumed a large value $\left(\sigma_{\ln M}=0.3\right)$ for scatter in the $\ln M$-richness relation in equation (35), and since we have assumed that $\Delta$ and cluster mass are maximally correlated. If the $\sigma_{\ln M}$ were reduced, or if $\Delta$ and mass are not maximally correlated, the tension between the model of equation (34) and the measured biases would increase. Note that if $\Delta$ and halo mass were maximally correlated, one could construct a zero-scatter mass proxy as some combination of $\lambda$ and $\Delta$; the implausibility of this scenario suggests that $\Delta$ and halo mass are almost certainly not maximally correlated.

\subsubsection{Calibration uncertainty in $\mathrm{b}(\mathrm{M})$}

Tinker et al. (2010) find scatter around the $b(M)$ measured across different cosmological simulations of roughly 6 per cent. Such scatter contributes a fundamental source of uncertainty to the massrichness constraints we derive using $b(M)$. We introduce this uncertainty into our model by multiplying $b(M)$ by $\left(1+b_{\text {scaling }}\right)$, where $b_{\text {scaling }}$ is a new parameter of our model. We place a Gaussian prior on $b_{\text {scaling }}$ centred at zero with $\sigma=0.06$. In principle, simply scaling $b(M)$ by $\left(1+b_{\text {scaling }}\right)$ may not fully capture the cross-simulation variation observed by Tinker et al. (2010). However, this is a reasonable approach given that we do not have probability distributions for all of the parameters of the Tinker et al. (2010) bias model. Correctly modelling the uncertainty in $b(M)$ will be an important part of future attempts to use cluster clustering to constrain massobservable relations. We discuss prospects for improving the $b(M)$ calibration in Section 9.

\subsection{Likelihood analysis}

We adopt a Gaussian likelihood for the data given the model:

$\mathcal{L}(\boldsymbol{d} \mid \boldsymbol{p}) \propto \exp \left[-\frac{1}{2}(\boldsymbol{d}-\boldsymbol{m}(\boldsymbol{p}))^{T} \mathbf{C}^{-1}(\boldsymbol{d}-\boldsymbol{m}(\boldsymbol{p}))\right]$,

where $\boldsymbol{d}$ is the data vector (containing all the cross-richness correlation function measurements) and $\boldsymbol{m}(\boldsymbol{p})$ is the model vector, which is a function of the parameter vector, $\boldsymbol{p}$. We compute $\mathbf{C}^{-1}$ using the estimator from Hartlap, Simon \& Schneider (2007):

$\widehat{\mathbf{C}^{-1}}=\frac{N-d-2}{N-1} \mathbf{C}^{-1}$,

where $N$ is the number of jackknife regions (in this case $N=800$ ), $d$ is the length of our data vector (in this case $d=80$ ) and $\mathbf{C}$ is the covariance matrix estimated from the jackknifing procedure.

We remind the reader that we perform two fits to the data: one in which the bias values themselves are the free parameters, and the other using a parametrized version of the mass-richness relationship and the $b(M)$ relation from Tinker et al. (2010). For reference, we reproduce here the complete model for the bias of the $\alpha$ th richness/redshift bin:

$$
\begin{aligned}
& b^{\alpha}=\frac{1}{N_{\alpha}} \sum_{i}^{N_{\alpha}}\left(c_{0}+c_{1} \Delta_{i}\right) b_{\text {scaling }} \\
& \times\left[(1-f) \int \mathrm{d} M b\left(M, z_{i}\right) P\left(M \mid \lambda_{i}, z_{i}\right)\right. \\
& \left.+f \int \mathrm{d} M_{1} b\left(M_{1}\left[1+g\left(\frac{1-q}{q}\right)^{\alpha}\right]\right) P\left(M_{1} \mid q \lambda_{i}, z_{i}\right)\right],
\end{aligned}
$$

where $P\left(M \mid \lambda_{i}, z_{i}\right)$ is given by equation (13). As stated above, we do not model the biases of the low-richness clusters $(5<\lambda<20)$ with equation (38), but instead treat the biases of each low-richness bin (one for each redshift bin) as a free parameter.

The analysis of Rozo \& Rykoff (2014) and Rozo et al. (2015a) suggests that the scatter in the mass-richness relationship is $\sigma_{\ln M} \sim 0.2-0.3$. In this analysis, we treat $\sigma_{\ln M}$ as a free parameter, imposing a flat prior $\sigma_{\ln M} \in[0.05,0.5]$. We find little degeneracy between $\sigma_{\ln M}$ and $A$ or $\alpha$. We marginalize over $\sigma_{\ln M}$ in the constraints presented below. The priors on all of our model parameters 
are summarized in Table 1. These priors, combined with the likelihood in equation (36), allow us to compute the posterior on the parameters of our model given the observed data. We use a Markov chain Monte Carlo approach to sample this multidimensional posterior. We perform the sampling using EMCEE (Foreman-Mackey et al. 2013). The entire analysis pipeline (from calculating $w_{M}(\theta)$ to sampling the posterior) is implemented in the $\operatorname{CosmoSIS}^{6}$ framework (Zuntz et al. 2015).

\subsection{Simulations}

In order to validate our approach to measuring the mass-richness relationship of redMaPPer clusters, we apply the analysis pipeline that we have developed to a mock data set generated from simulations. We use an $N$-body simulation of a flat $\Lambda \mathrm{CDM}$ cosmological model with $\Omega_{\mathrm{m}}=0.286, h_{0}=0.7, \Omega_{\mathrm{b}}=0.047, n_{\mathrm{s}}=0.96$ and $A_{\mathrm{s}}=2.1 \times 10^{-9}$, run with $1400^{3}$ particles in a box $1050 \mathrm{Mpc} h^{-1}$ on a side, with the L-GADGET code, a variant of GADGET (Springel 2005). A lightcone was output from the simulation on the fly, to produce a quarter-sky simulation over the same redshift range as the data. We use a halo catalogue generated by the ROCKSTAR halo finder (Behroozi, Wechsler \& Wu 2013), run directly on the dark matter lightcone.

The probability that a halo of mass $M$ has richness $\lambda, P(\lambda \mid M)$, can be related to our parametrized $P(M \mid \lambda)$ using Bayes' theorem: $P(\lambda \mid M) \propto P(M \mid \lambda) P(\lambda)$. The mass-richness relation $P(M \mid \lambda)$ is given by equations (13) and (14). We determine the prior $P(\lambda)$ from the real data by fitting a Schechter function to the observed distribution of $\lambda$. We randomly draw from $P(\lambda \mid M)$ to generate a simulated value of $\lambda$ for each mock halo.

We choose the parameters $A, \alpha$ and $\beta$ of the mass-richness relation to ensure that the distribution of simulated clusters in richness and redshift is close to the distribution of real clusters. Since the cosmological model used to generate the simulation used herein is slightly different from the currently favoured $\Lambda \mathrm{CDM}$ cosmological model, it stands to reason that matching the observed cluster abundance will require somewhat different mass-richness parameters than are obtained from our analysis of the data. We find that setting $\ln \left(A / \mathrm{M}_{\odot}\right)=33.15, \alpha=1.0, \beta=1.0$ and $\sigma_{\ln M}=0.2$ results in a cluster catalogue that has roughly the same number density of clusters as the SDSS data.

The simulated redMaPPer catalogue does not include the effects of line-of-sight projections of clusters, because we use a pure halo catalogue generated using the three-dimensional positions of the dark matter particles. As a result, two haloes that happen to be close together on the sky will never be lumped into the same simulated redMaPPer object. For this reason, when analysing the simulation data, we fix $f=0$. Furthermore, while the simulations include the effects of assembly bias in the haloes, without a more comprehensive modelling approach, we do not know an appropriate value of $\Delta$ to assign to each cluster. We therefore fix $\langle\Delta\rangle_{M, z}=0$ and $c_{1}=0$ when analysing the simulated redMaPPer catalogue.

We apply the same analysis pipeline to the simulated data as to the real data. This includes the process of pair counting, jackknifing to determine the covariance matrix and fitting for the mass-richness parameters. When computing $w_{\mathrm{M}}(\theta)$ for the analysis of the simulated data, we use the same cosmological model that was used to generate the $N$-body simulations.

Fig. 5 shows the constraints obtained on the mass-richness parameters by applying our pipeline to the simulated data. The input

\footnotetext{
${ }^{6}$ https://bitbucket.org/joezuntz/cosmosis/wiki/Home
}

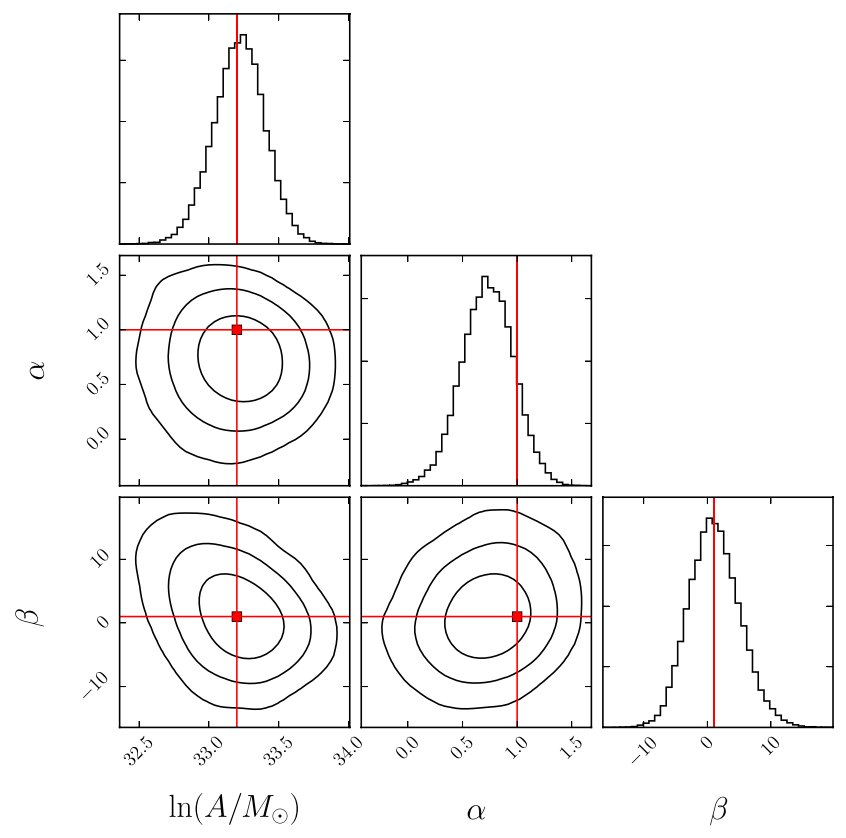

Figure 5. Posteriors on the parameters of the mass-richness relation resulting from the analysis of a simulated cluster catalogue generated from $\mathrm{N}$-body simulations. Contour lines enclose 68 per cent, 95 per cent and 99.7 per cent of the probability density. Input values are shown by the red lines. Our analysis successfully recovers the input parameters of the mass-richness relation.

mass-richness parameters are shown as the red lines, and contour lines represent $1 \sigma, 2 \sigma$, etc. As evidenced by the figure, our analysis appears to correctly extract the input model parameters. We find $\chi^{2} /$ d.o.f. $=70.7 / 73$, suggesting that the model is an excellent fit to the data. Furthermore, we find that the error bars on the extracted model parameters are similar to those found in our analysis of the real data.

\section{RESULTS}

Figures showing the measured angular correlation functions and the best-fitting models are collected in Appendix A, Figs A1 and A2. The best-fitting models for the bias-only parametrization are shown in red, while the best-fitting mass-richness models are shown in green. The results of these two fits are very similar (in many cases the two curves are indistinguishable by eye). This suggests that the mass-richness parametrization is well matched to the true clustering biases of the redMaPPer clusters.

We find somewhat low values of $\chi^{2}$, with $\chi^{2} /$ d.o.f. $=57.1 / 72$ for the bias-only model, and $\chi^{2} /$ d.o.f. $=57.5 / 75$ for the massrichness model. ${ }^{7}$ The probabilities for $\chi^{2}$ to be less than the quoted values are roughly 10 per cent and 6 per cent, respectively. We caution, however, that since the covariance matrix is estimated using a jackknife approach, there are noise fluctuations in the covariance matrix that can increase or reduce the recovered scatter. Roughly speaking, if our error bars on the correlation function measurements were reduced by $\sim 10$ per cent, we would have $\chi^{2} /$ d.o.f. $\sim 1$. As we show below, our error bars on the parameters of the mass-richness relation are dominated by uncertainty in the model parameters. A

\footnotetext{
${ }^{7}$ When calculating the d.o.f. for the mass-richness model, we do not include the various systematic parameters that have small impacts on our model predictions.
} 
Table 2. Marginalized constraints on the biases of redMaPPer clusters in bins of richness and redshift.

\begin{tabular}{lcc}
\hline Richness range & $0.1<z<0.26$ & $0.26<z<0.33$ \\
\hline $5<\lambda<20$ & $b=1.85 \pm 0.06$ & $b=1.74 \pm 0.05$ \\
$20<\lambda<28$ & $b=2.8 \pm 0.2$ & $b=3.0 \pm 0.2$ \\
$28<\lambda<41$ & $b=3.1 \pm 0.3$ & $b=3.7 \pm 0.3$ \\
$41<\lambda<\infty$ & $b=4.6 \pm 0.3$ & $b=5.1 \pm 0.4$ \\
\hline
\end{tabular}

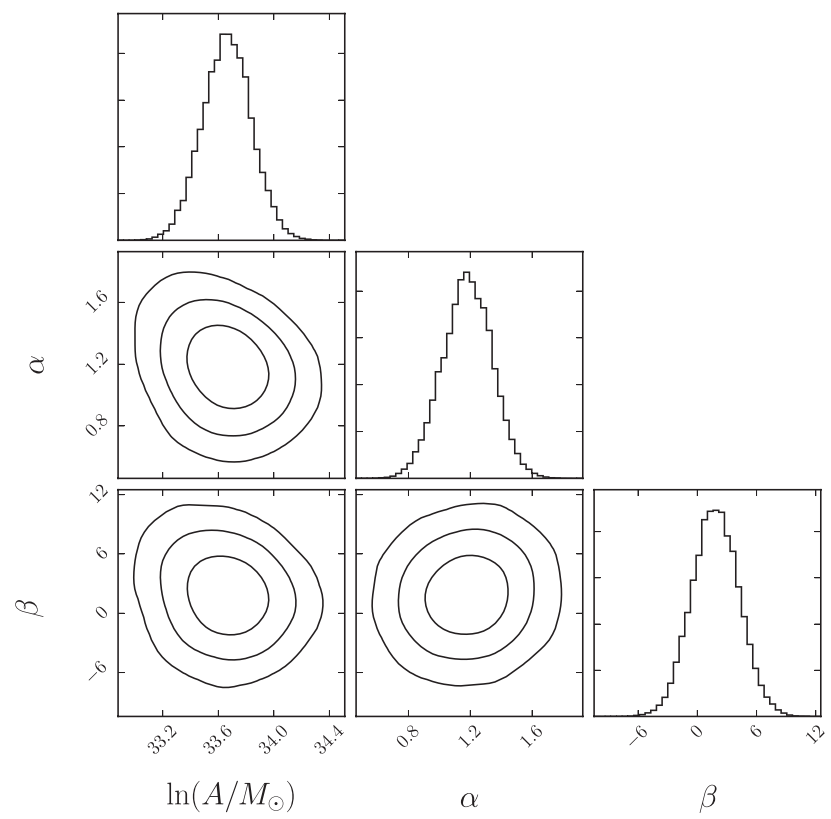

Figure 6. Constraints on the parameters of the mass-richness relationship obtained from a fit to the measured angular correlation functions. Contour lines enclose 68 per cent, 95 per cent and 99.7 per cent of the probability density.

10 percent reduction in the measurement errors would therefore have little impact on our results.

Table 2 summarizes the constraints on the clustering biases for each individual richness/redshift bin. As expected, the bias increases steadily with increasing richness.

Fig. 6 shows the constraints on the parameters of the mass-richness relation derived from the angular clustering measurements. $^{8}$ The value of $\ln A$ - the amplitude parameter of the mass-richness relationship - is constrained to be $\ln \left(A / \mathrm{M}_{\odot}\right)=33.66 \pm 0.18$, corresponding to an 18 per cent mass constraint. The slope of the mass-richness relationship is constrained to be $\alpha=1.18 \pm 0.16$. The scaling with redshift is constrained to be $\beta=1.86 \pm 2.4$; the weakness of this constraint is due in part to our use of only two redshift bins and the fact that our cluster sample is narrowly distributed in redshift. These constraints are summarized in the 'Posterior' column of Table 1.

Our calibration can be compared to the weak lensing mass estimator of S16. We emphasize that despite common authors between this work and S16, no comparison between the two analyses was performed before the analyses pipelines were finalized. Our decision to focus on the NGC exclusively did come after a comparison with S16 had occurred, though we believe that we are well justified in our choice.

${ }^{8}$ Contour plots were made using CORNER.PY (Foreman-Mackey et al. 2016).
S16 provide the current best weak lensing mass calibration of redMaPPer clusters, and they discuss in detail how their mass calibration compares to others in the literature. While the constraints on the slope of the mass-richness relation are in good agreement between the two works, there is mild tension on the recovered amplitude assuming our fiducial cosmology, with the clusteringderived constraints preferring a somewhat higher amplitude. At the richness pivot point used in this work, $\lambda_{0}=35$, S16 predict $\log _{10}\left(M / \mathrm{M}_{\odot}\right)=14.43 \pm 0.04$, while our constraints yield $\log _{10}\left(M / \mathrm{M}_{\odot}\right)=14.62 \pm 0.08$; this discrepancy corresponds to roughly $2.2 \sigma$ tension. Because the clustering analysis prefers a slightly lower value of $\alpha$ than the weak lensing analysis, this tension is reduced for $\lambda>35$. For $\lambda<35$, the tension remains roughly the same since the error bars increase as one moves away from the $\lambda$ pivot point. We postpone a discussion of the origin of this tension, and a more detailed comparison of the two results, to future work. That is, our current results reflect our best understanding of both the weak lensing and clustering analysis prior to the comparison of the two works.

We have also considered a power-law bias-richness relation as per equation (10). This model has five parameters: $A_{\lambda}, \alpha_{\lambda}, \beta_{\lambda}$ and two bias parameters for $5<\lambda<20$ clusters in the two redshift bins. Fitting this model to the data, we find $A_{\lambda}=3.54 \pm 0.13, \alpha_{\lambda}=0.57$ \pm 0.08 and $\beta_{\lambda}=2.0 \pm 1.1$ with $\chi^{2} /$ d.o.f. $=57.7 / 75$.

\section{CHARACTERIZING THE UNCERTAINTY IN THE MASS-RICHNESS RELATION}

The uncertainties on the mass-richness parameters presented in Fig. 6 and Table 1 receive contributions from several sources: statistical noise in the measurement of the angular correlation functions, uncertainty on the parameters of the line-of-sight projection model, uncertainty on the parameters of the assembly bias model and scatter around the $b(M)$ relation from Tinker et al. (2010). In addition to these sources of scatter that are explicitly included in our model, it is also possible that our measurement is affected by systematic biases that we have not modelled. Below, we determine the approximate contributions of both modelled sources of scatter and unmodelled systematic errors to the uncertainty budget of our parameter constraints.

\subsection{Statistical noise in the correlation function measurement}

We first investigate how statistical noise in the measurement of $w^{\alpha \beta}(\theta)$ contributes to our parameter uncertainties. The contribution of statistical noise to our parameter uncertainties can be determined by setting the parameters of the line-of-sight projection model, the parameters of the assembly bias model and $b_{\text {scaling }}$ to constant values rather than allowing these parameters to vary. Any uncertainty in our parameter constraints that remains after fixing these model parameters must be due to measurement noise alone.

For the purposes of this test, we fix $f=0.1, q=0.75, g=0.5$, $\langle\Delta\rangle_{M, z}=0.032, c_{1}=3.6$ and $b_{\text {scaling }}=0$, corresponding to the central values of the priors on these parameters. The results of this section are relatively insensitive to the precise values that we choose here. Upon re-fitting this constrained model to the data, we find that the posterior on $\ln A$ is significantly tighter than that obtained in our fiducial analysis. In other words, the uncertainty on the amplitude of the mass-richness relation is dominated by uncertainty on the model parameters that we marginalize over. In the absence of uncertainty on these model parameters, the error on $\ln A$ would be only $\sim 0.07$ instead of $\sim 0.18$. Statistical noise 
in the measurement of $w^{\alpha \beta}(\theta)$ contributes only $\sim 10$ per cent of the variance of our baseline constraint on $\ln A$.

On the other hand, we find that statistical measurement error contributes $\sim 70$ percent of the variance of our constraints on $\alpha$ and $\beta$. The uncertainties on these parameters would be significantly reduced with a larger cluster catalogue distributed over a wider redshift range.

\subsection{Scatter around $b(M)$ from Tinker et al. (2010)}

Our fiducial analysis assumes a scatter of 6 per cent around the $b(M)$ model from Tinker et al. (2010), parametrized with $b_{\text {scaling. To }}$ measure the contribution of this source of scatter to our parameter uncertainties, we consider a model that allows $b_{\text {scaling }}$ to vary with the fiducial prior, but that fixes all other model parameters to the values given in Section 7.1. Comparing the posteriors obtained from fitting this model to the data to those obtained in Section 7.1 provides a measure of the contribution of uncertainty on $b_{\text {scaling }}$ to our final parameter uncertainties.

We find that uncertainty on $b_{\text {scaling }}$ dominates the uncertainty on $\ln A$, contributing roughly 60 per cent of the variance of our fiducial constraint on this parameter. In other words, our constraint on the normalization of the mass-richness relation for redMaPPer clusters is currently limited by uncertainty in the $b(M)$ relation. In contrast, the posteriors on $\alpha$ and $\beta$ are less affected by uncertainty on $b_{\text {scaling; }}$; uncertainty on $b_{\text {scaling }}$ contributes only $\sim 30$ per cent of the variance of our constraints on these parameters.

\subsection{Uncertainty on assembly bias parameters}

To evaluate the contribution of uncertainty on the assembly bias parameters $c_{1}$ and $\langle\Delta\rangle_{M, z}$ to our constraints on the mass-richness parameters, we fit a model to the data that allows the assembly bias parameters to vary over their fiducial priors, but that keeps the other model parameters fixed to the values in Section 7.1. We find that uncertainty on the assembly bias parameters contributes roughly 30 percent of the variance of our baseline constraint on $\ln A$, but contributes negligibly to the variance of the constraints on $\alpha$ and $\beta$. Uncertainty on the assembly bias parameters therefore contributes a level of uncertainty on the normalization of the mass-richness relation that is greater than that of measurement uncertainty. Note that these results assume that the dependence of redMaPPer clustering on $\Delta$ measured here and by M16 is due to a form of assembly bias.

We also evaluate how much the inclusion of assembly bias effects due to $\Delta$ shifts our parameter constraints relative to a model that does not include these effects. We fit the data with a model that has $\langle\Delta\rangle_{M, z}=0.0$ and $c_{1}=0$, but otherwise is identical to the baseline analysis. The results of this fit suggest that the inclusion of assembly bias due to $\Delta$ in our analysis causes our best-fitting $\ln A$ to increase by roughly 0.15 , corresponding to $0.8 \sigma$. The direction of this shift is as anticipated in Section 5.3. The inclusion of assembly bias in our analysis has a smaller impact on the best-fitting values of $\alpha$ and $\beta$.

\subsection{Uncertainty on line-of-sight projection parameters}

We follow a similar approach to that outlined in Section 7.3 to estimate the contribution of uncertainty on the line-of-sight projection parameters $f, q$ and $g$ to our mass-richness parameter uncertainties. We fit a model to the data that allows the projection parameters to vary over the priors in Table 1, but which has the assembly bias and $b_{\text {scaling }}$ parameters fixed to the same values as in Section 7.1. Comparing the parameter uncertainties obtained using this constrained model to the parameter uncertainties obtained in Section 7.1 suggests that uncertainty on the projection parameters contributes less than 10 per cent of the variance of $\ln A, \alpha$ and $\beta$.

We can also ask how much line-of-sight projections shift our parameter constraints relative to an analysis that does not account for projections. To do this, we fit a model to the data that has $f=0.0$, but otherwise is identical to the baseline model. Analysing the data with this model reveals that including line-of-sight projections in our analysis shifts the maximum likelihood values of $\ln A, \alpha$ and $\beta$ by less than $0.1 \sigma$.

\subsection{Systematics in $w^{\alpha \beta}(\theta)$ measurement}

As described in Section 4.2, we apply weights to the redMaPPer random catalogue to correct for an observed dependence of the cluster density on the $g$-band sky flux. A similar variation in the cluster density is also observed with the $E(B-V)$ dust extinction maps from Schlegel et al. (1998, see Fig. 2). To determine the level of systematic error introduced into our measurements by only correcting for the correlation between cluster density and $g$-band sky flux, we repeat the correlation function measurement and fitting using a random catalogue weighted instead by $E(B-V)$ dust extinction.

We find that the application of the $E(B-V)$ weights causes our best-fitting $\ln A, \alpha$ and $\beta$ to change by less than $0.05 \sigma$. This suggests that the systematic error introduced by only correcting for variations in cluster density with $i$-band PSF is small compared to our error bars.

As mentioned above, the results presented thus far were derived using only the NGC region of SDSS. We have also applied our clustering analysis to the SGC region. We find some tension between the clustering measurements in both regions, with the SGC preferring somewhat higher values of $\ln A$ and somewhat lower values of $\alpha$ than the NGC. Considering the bias-only parametrization, we find the amplitude of the bias-mass relation to be consistent between the two regions. However, the constraints on $\alpha_{\lambda}$ for the two regions differ by $2.1 \sigma$. There is also roughly $2.4 \sigma$ tension in the biases of the clusters in the richness/redshift bin $5<\lambda<20,0.18<z<0.26$ between the two regions. There is no significant tension in the $\beta_{\lambda}$ constraints between the two regions. When considering constraints on the mass-richness parametrization, the tension between NGC and SGC is reduced because the marginalization over $b_{\text {scaling }}$ and the assembly bias parameters effectively increase the error bars on the parameters. Tension in the $\ln A$ constraints from the two regions is only $0.3 \sigma$, while tension on $\alpha$ is $2 \sigma$.

If the clustering of redMaPPer clusters is different in the NGC and the SGC, one might expect the abundance of redMaPPer clusters to also be different in the NGC and the SGC. Fig. 7 shows a comparison of the cluster density as a function of richness between the NGC and the SGC. The error bars in the figure only include the Poisson contribution and not sample variance. In general, the agreement between the two regions with respect to cluster abundance appears to be quite good. However, we find $\chi^{2} /$ d.o.f. $=43.7 / 26$ for the low-redshift bin. The high value of $\chi^{2}$ is driven almost entirely by clusters with $\lambda \sim 10$. For the high-redshift bin, we find $\chi^{2} /$ d.o.f. $=34.8 / 26$. Note, though, that by only including the Poisson contribution to the error bars, we have overestimated the tension between the NGC and SGC in this comparison.

In summary, we find some evidence for tension between the clustering-derived parameter constraints in the NGC and SGC. This tension is most pronounced for our constraints on $\alpha$ : the NGC 


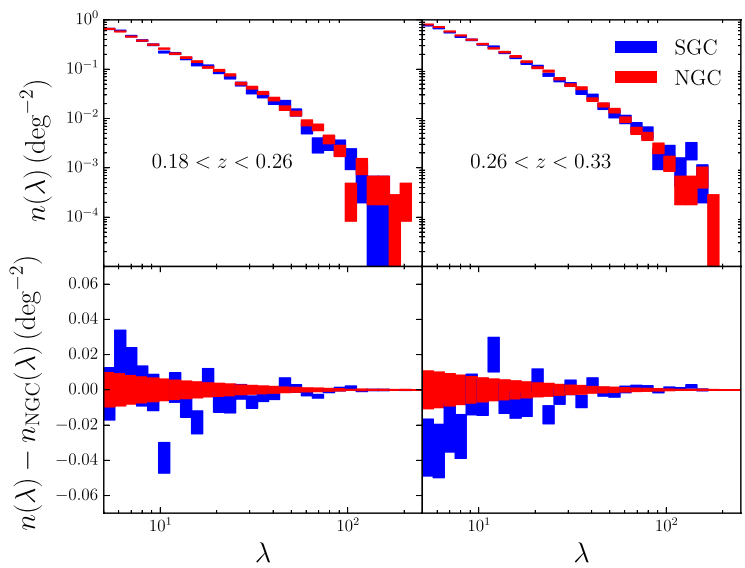

Figure 7. Cluster density as a function of richness for the NGC and SGC regions. We find some evidence for tension in the abundance of clusters between the NGC and SGC; this tension is driven by low-redshift clusters with $\lambda \sim 10$.

prefers a higher value of $\alpha$ than the SGC by roughly $2 \sigma$. Tension between the two regions with respect to the other mass-richness parameters is consistent with noise. There is also some evidence for tension in the abundance of low-redshift, $\lambda \sim 10$ clusters between the two regions. We note that Tojeiro et al. (2014) also found evidence for tension between the NGC and SGC at low redshift $(z<0.25)$ in their measurements of the clustering of galaxies identified in SDSS. Since the origin of this tension is not well understood at this time, we refrain from combining the parameter constraints obtained from the NGC and SGC, and instead present only the results for the NGC. This choice also has the advantage that it makes comparison to the weak lensing mass constraints from S16 more straightforward since the S16 analysis was only applied to clusters in the NGC.

\subsection{Jackknife covariance estimation}

Our jackknife estimate of the covariance matrix does not show any obvious evidence for systematic errors. However, we feel it necessary to confirm that our covariance estimate is robust with respect to the choice of jackknife regions. To do this, we repeat the jackknifing procedure with 10 per cent fewer jackknife regions (720 instead of $\sim 800$ ). Re-fitting the correlation function measurements for the mass-richness parameters using the modified covariance estimate leads to small shift in the best-fitting mass-richness parameters, all well within the error bars. The largest shift observed is in the best-fitting value of $\alpha$, which changes by $0.23 \sigma$. Given the smallness of these shifts compared to the error bars, any uncertainty in our mass-richness constraints introduced by the choice of jackknife regions can be safely ignored.

\section{COSMOLOGY DEPENDENCE}

The results presented above have assumed a fixed cosmological model. In this section, we vary the cosmological parameters and re-fit the data to determine how the mass-richness relation varies with cosmology. We focus here on $\Omega_{\mathrm{m}}, A_{\mathrm{s}}$ and $h_{0}$. Other cosmological parameters are expected to have a subdominant effect on the predicted correlation function.

We compute the maximum likelihood mass-richness parameters at 12 points in $\left(\Omega_{\mathrm{m}}, A_{\mathrm{s}}, h\right)$ parameter space. These points are chosen to be within 10 per cent of the fiducial values of $\Omega_{\mathrm{m}}, A_{\mathrm{s}}$ and $h_{0}$ since Planck Collaboration XIII (2015b) constrain these parameters to better than 10 per cent. Furthermore, restricting $\Omega_{\mathrm{m}}$, $A_{\mathrm{s}}$ and $h_{0}$ to a narrow interval ensures that the dependence of the mass-richness relation on these parameters is close to linear over the sampled range. For each point $\left(\Omega_{\mathrm{m}}, A_{\mathrm{s}}, h\right)$, we determine the maximum likelihood values of $\ln A, \alpha$ and $\beta$ given our correlation function measurements. Using linear regression, we then compute the dependence of $\ln A, \alpha$ and $\beta$ on $\Omega_{\mathrm{m}}, A_{\mathrm{s}}$ and $h_{0}$.

For the amplitude parameter, $\ln A$, we find a best-fitting linear model:

$$
\ln \left(A / \mathrm{M}_{\odot}\right)=33.68+2.9 \delta \Omega_{\mathrm{m}}+1.0 \delta A_{\mathrm{s}}+1.8 \delta h,
$$

where we have defined

$\delta \Omega_{\mathrm{m}}=\frac{\Omega_{\mathrm{m}}-0.3089}{0.3089}$

$\delta A_{\mathrm{s}}=\frac{A_{\mathrm{s}}-2.141 \times 10^{-9}}{2.141 \times 10^{-9}}$

$\delta h=\frac{h-0.6774}{0.6774}$

as the fractional departures of $\Omega_{\mathrm{m}}$ and $A_{\mathrm{s}}$ from the values assumed in our baseline analysis. We find that 10 per cent changes in $\Omega_{\mathrm{m}}$, $A_{\mathrm{s}}$ and $h_{0}$ result in $1.6 \sigma, 0.6 \sigma$ and $1.0 \sigma$ shifts in $\ln A$, respectively. Note that $\Omega_{\mathrm{m}}, A_{\mathrm{s}}$ and $h_{0}$ are constrained to $\sim 2$ per cent by Planck Collaboration XIII (2015b); the variation in $\ln A$ as a function of $\Omega_{\mathrm{m}}, A_{\mathrm{s}}$ and $h_{0}$ over the range allowed by Planck is therefore below the uncertainty on $\ln A$.

We find that $\alpha$ is most sensitive to $\Omega_{\mathrm{m}}$ and $h_{0}$, with no significantly measured dependence on $A_{\mathrm{s}}$ over the range of $A_{\mathrm{s}}$ values considered. Our best-fitting relation is

$\alpha=1.17-0.6 \delta \Omega_{\mathrm{m}}-0.5 \delta h$.

Changing $\Omega_{\mathrm{m}}$ and $h_{0}$ by 10 per cent therefore changes $\alpha$ by $0.4 \sigma$ and $0.3 \sigma$, respectively. Any dependence of $\alpha$ on $A_{\mathrm{s}}$ is significantly below our error bar on $\alpha$.

We find that the $\beta$ is most sensitive to $h_{0}$, with a best-fitting linear relationship described by

$\beta=1.8+1.1 \delta_{h}$.

A 10 per cent change in $h_{0}$ therefore results in a $0.05 \sigma$ change to $\beta$. The dependence of $\beta$ on $\Omega_{\mathrm{m}}$ and $A_{\mathrm{s}}$ is well below our error bar on $\beta$ and we do not report it here.

\section{DISCUSSION}

We have measured the angular correlation function of redMaPPer clusters identified in SDSS data. By fitting models to these measurements, we have extracted constraints on several parameters describing the clustering biases and mass-richness relationship of redMaPPer clusters. Our constraints on the biases of redMaPPer clusters in bins of richness and redshift are shown in Table 2. Our constraints on the mass-richness relationship for redMaPPer clusters are shown in Fig. 6 and summarized in Table 1.

We measured the correlation between clustering bias and $\Delta$, where $\Delta$ is related to the concentration of member galaxies and is defined in equation (22). These measurements support the results of M16 and More et al. (2016). Expanding on those works, we have also measured the slope of the bias- $\Delta$ relationship. Furthermore, 
our clustering measurements provide evidence that the dependence of redMaPPer clustering on $\Delta$ is not due to dependence of $\Delta$ on cluster mass, independent of the weak lensing measurements of M16. Even if one assumes that $\Delta$ and mass are maximally correlated, the clustering measurements shown in Fig. 4 cannot be explained without invoking assembly bias. Finally, we find that including assembly bias due to $\bar{R}_{\text {mem }}$ in our analysis increases the best-fitting amplitude of the mass-richness relationship by roughly 15 per cent, reflecting the fact that the signal seen by M16 is significantly larger than was expected a priori. This measured increase in the best-fitting $\ln A$ assumes that the measured dependence of redMaPPer clustering on $\bar{R}_{\text {mem }}$ is a form of assembly bias, as claimed by M16. If the dependence of redMaPPer clustering on $\bar{R}_{\text {mem }}$ were instead due to an environmental or selection effect, for instance, the true shift in $\ln A$ due to assembly bias effects need not be so large.

In the absence of systematic sources of uncertainty, our analysis of redMaPPer clustering constrains the amplitude of the massrichness relation, $A=\exp (\ln A)$, to 7 per cent. Including systematic sources of uncertainty degrades our constraint on $A$ to 19 per cent precision. The dominant source of systematic uncertainty is scatter around the $b(M)$ relation from Tinker et al. (2010), which contributes roughly 60 per cent of the variance of our final constraint on $A$. If the uncertainty on $b(M)$ were significantly reduced (e.g. by using very large volume simulations to calibrate $b(M)$ ), the error bar on $A$ could be reduced to 11 per cent, roughly the same level of uncertainty obtained by the weak lensing mass calibration of S16. Uncertainty on the assembly bias parameters $c_{0}$ and $\langle\Delta\rangle_{M, z}$ is the next largest source of systematic error on $A$. Assuming that the observed dependence of redMaPPer clustering on $\Delta$ is due to assembly bias, uncertainty on $c_{0}$ and $\langle\Delta\rangle_{M, z}$ makes a contribution to the variance of $\ln A$ that is comparable to that of statistical noise. We find that line-of-sight projection effects have a negligible impact on our parameter constraints. Our constraints on the slope parameters of the mass-richness relation $-\alpha$ and $\beta$ - are not as strongly affected by systematic uncertainties and are currently statistics limited.

We emphasize that future measurements of cluster clustering with e.g. the Dark Energy Survey can significantly reduce the statistical uncertainty on the measured correlation functions, and enable the assembly bias parameters to be measured more tightly. In particular, we note that the larger the volume probed, the lower the errors, so cluster clustering is particularly well suited for calibrating the mass-richness relation of high-redshift clusters, exactly where the statistical and systematic uncertainty in weak lensing mass calibration is greatest. Critically, the current errors in the recovered mass-richness relation are systematic dominated, with the dominant systematic being theoretical uncertainty in the calibration of the bias-mass relation of dark matter haloes. Thus, unlike weak lensing mass calibration efforts, a path towards improved mass calibration from cluster clustering is very straightforward: one just needs to implement a simulation programme geared towards improving the calibration of halo bias. The prospect of improved data sets, reduced statistical errors and a straightforward path towards reducing systematic uncertainties all bode well for the future of cluster clustering as a method for calibrating the mass-richness relation of galaxy clusters.

\section{ACKNOWLEDGEMENTS}

This work received partial support from the US Department of Energy under contract number DE-AC02-76SF00515 and from the National Science Foundation under NSF-AST-1211838. EB and BJ are partially supported by the US Department of Energy grant
DE-SC0007901. We thank Matthew Becker and Michael Busha for their contributions to the $N$-body simulations used in this work, and Yao-Yuan Mao for helpful discussions about assembly bias. We also thank Mike Jarvis for developing TREECORR and for help with running it. We thank Melanie Simet, Rachel Mandelbaum and Gary Bernstein for useful discussions related to this work.

\section{REFERENCES}

Aihara H. et al., 2011, ApJS, 193, 29

Albrecht A. et al., 2006, preprint (arXiv:astro-ph/0609591)

Angulo R. E., Springel V., White S. D. M., Jenkins A., Baugh C. M., Frenk C. S., 2012, MNRAS, 426, 2046

Baxter E. J., Rozo E., 2013, ApJ, 779, 62

Behroozi P. S., Wechsler R. H., Wu H.-Y., 2013, ApJ, 762, 109

Benson B. A. et al., 2013, ApJ, 763, 147

Boylan-Kolchin M., Springel V., White S. D. M., Jenkins A., 2010, MNRAS, 406, 896

Cooray A., Sheth R., 2002, Phys. Rep., 372, 1

Dalal N., White M., Bond J. R., Shirokov A., 2008, ApJ, 687, 12

Dawson K. S. et al., 2013, AJ, 145, 10

Foreman-Mackey D., Hogg D. W., Lang D., Goodman J., 2013, PASP, 125, 306

Foreman-Mackey D. et al., 2016, corner.py: corner.py v1.0.2, Available at http://dx.doi.org/10.5281/zenodo.45906

Gao L., Springel V., White S. D. M., 2005, MNRAS, 363, L66

Hartlap J., Simon P., Schneider P., 2007, A\&A, 464, 399

Hayashi E., White S. D. M., 2008, MNRAS, 388, 2

Holder G., Kosowsky A., 2004, ApJ, 616, 8

Hu W., Cohn J. D., 2006, Phys. Rev. D, 73, 067301

Huterer D. et al., 2015, Astropart. Phys., 63, 23

Jarvis M., Bernstein G., Jain B., 2004, MNRAS, 352, 338

Jing Y. P., Zhang P., Lin W. P., Gao L., Springel V., 2006, ApJ, 640, L119

Jing Y. P., Suto Y., Mo H. J., 2007, ApJ, 657, 664

Landy S. D., Szalay A. S., 1993, ApJ, 412, 64

Lewis A., Challinor A., Lasenby A., 2000, ApJ, 538, 473

Lima M., Hu W., 2004, Phys. Rev. D, 70, 043504

Limber D. N., 1953, ApJ, 117, 134

Majumdar S., Mohr J. J., 2003, ApJ, 585, 603

Mantz A. B. et al., 2015, MNRAS, 446, 2205

Mao Y.-Y., Williamson M., Wechsler R. H., 2015, ApJ, 810, 21

Miyatake H., More S., Takada M., Spergel D. N., Mandelbaum R., Rykoff E. S., Rozo E., 2016, Phys. Rev. Lett., 116, 041301 (M16)

More S. et al., 2016, ApJ, 825, 39

Norberg P., Baugh C. M., Gaztañaga E., Croton D. J., 2009, MNRAS, 396, 19

Planck Collaboration XXIV 2015a, preprint (arXiv:1502.01597)

Planck Collaboration XIII 2015b, preprint (arXiv:1502.01589)

Planck Collaboration XXVII 2015c, preprint (arXiv:1502.01598)

Rozo E., Rykoff E. S., 2014, ApJ, 783, 80

Rozo E. et al., 2010, ApJ, 708, 645

Rozo E., Rykoff E. S., Bartlett J. G., Evrard A., 2014, MNRAS, 438, 49

Rozo E., Rykoff E. S., Bartlett J. G., Melin J.-B., 2015a, MNRAS, 450, 592

Rozo E., Rykoff E. S., Becker M., Reddick R. M., Wechsler R. H., 2015b, MNRAS, 453, 38

Rykoff E. S. et al., 2014, ApJ, 785, 104

Schlegel D. J., Finkbeiner D. P., Davis M., 1998, ApJ, 500, 525

Sehgal N. et al., 2011, ApJ, 732, 44

Sereno M., Ettori S., 2015, MNRAS, 450, 3633

Simet M., McClintock T., Mandelbaum R., Rozo E., Rykoff E., Sheldon E., Wechsler R. H., 2016, preprint (arXiv:1603.06953) (S16)

Smith R. E. et al., 2003, MNRAS, 341, 1311

Springel V., 2005, MNRAS, 364, 1105

Takahashi R., Sato M., Nishimichi T., Taruya A., Oguri M., 2012, ApJ, 761, 152 
Tinker J. L., Robertson B. E., Kravtsov A. V., Klypin A., Warren M. S.,

Yepes G., Gottlöber S., 2010, ApJ, 724, 878

Tojeiro R. et al., 2014, MNRAS, 440, 2222

van Daalen M. P., Schaye J., Booth C. M., Dalla Vecchia C., 2011, MNRAS,

415, 3649

Vikhlinin A. et al., 2009, ApJ, 692, 1060

von der Linden A. et al., 2014, MNRAS, 439, 2

Wechsler R. H., Zentner A. R., Bullock J. S., Kravtsov A. V., Allgood B., 2006, ApJ, 652, 71

Weinberg D. H., Mortonson M. J., Eisenstein D. J., Hirata C., Riess A. G., Rozo E., 2013, Phys. Rep., 530, 87

Wu H.-Y., Rozo E., Wechsler R. H., 2008, ApJ, 688, 729

York D. G. et al., 2000, AJ, 120, 1579

Zentner A. R., Berlind A. A., Bullock J. S., Kravtsov A. V., Wechsler R. H., 2005, ApJ, 624, 505
Zu Y., Weinberg D. H., Rozo E., Sheldon E. S., Tinker J. L., Becker M. R., 2014, MNRAS, 439, 1628

Zuntz J. et al., 2015, Astron. Comput., 12, 45

\section{APPENDIX A: MEASUREMENTS AND BEST-FITTING MODELS}

Figs A1 and A2 show the angular correlation function for redMaPPer clusters for two different redshift bins, and our best-fitting model both when adopting a free bias parameter for each richness bin (red) and when modelling the mass-richness relation (green).

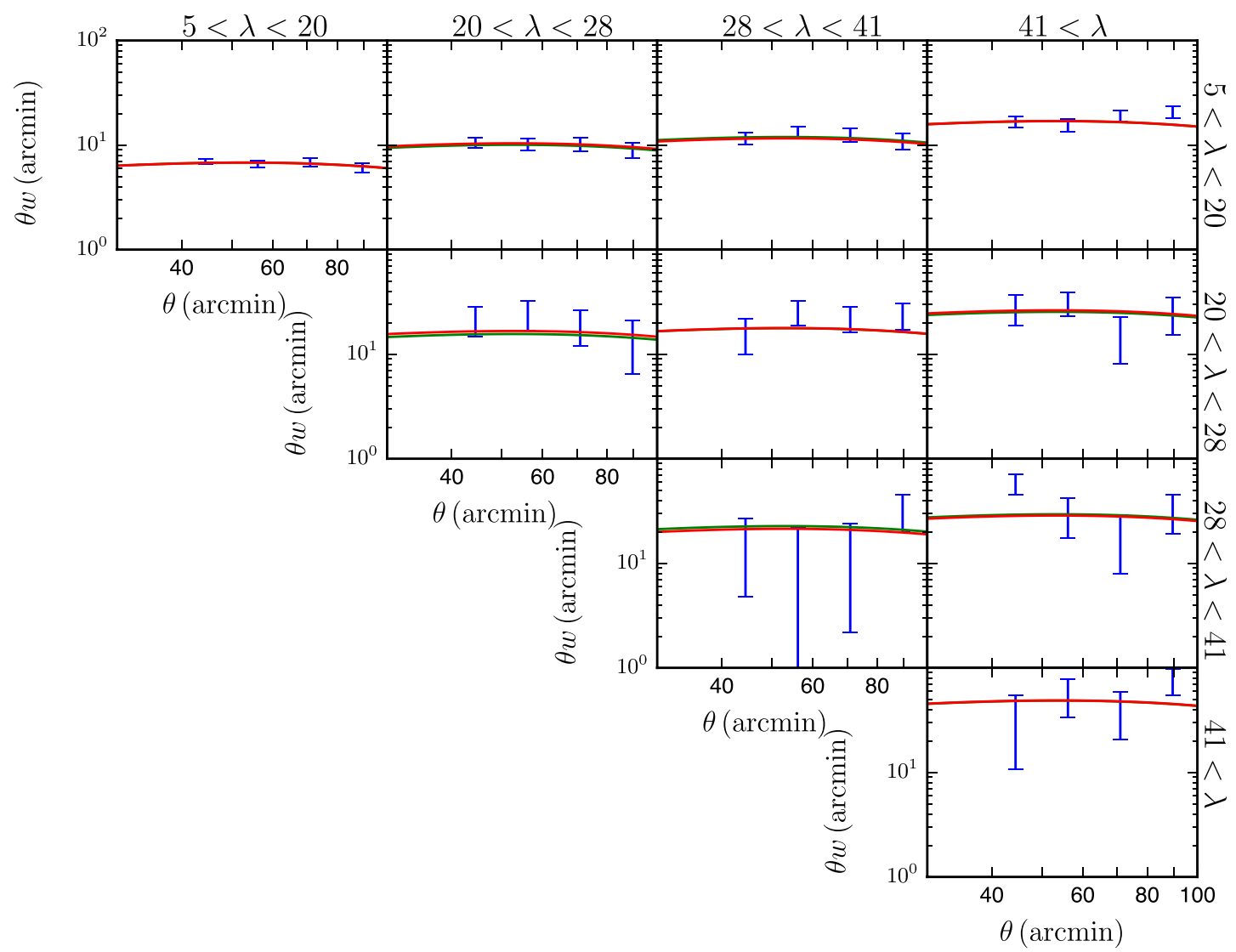

Figure A1. The angular cross-correlation function, $\theta w^{\alpha \beta}(\theta)$, between redMaPPer clusters in several different richness bins for the redshift interval $0.18<z<0.26$. The richness bins are indicated on the top and right sides of the figure. Error bars are the diagonal elements of the covariance matrix. The red curves show the best-fitting model when each richness bin is assigned a free bias parameter, while the green curves are obtained through modelling of the mass-richness relation (in many cases, the green and red curves are indistinguishable). 


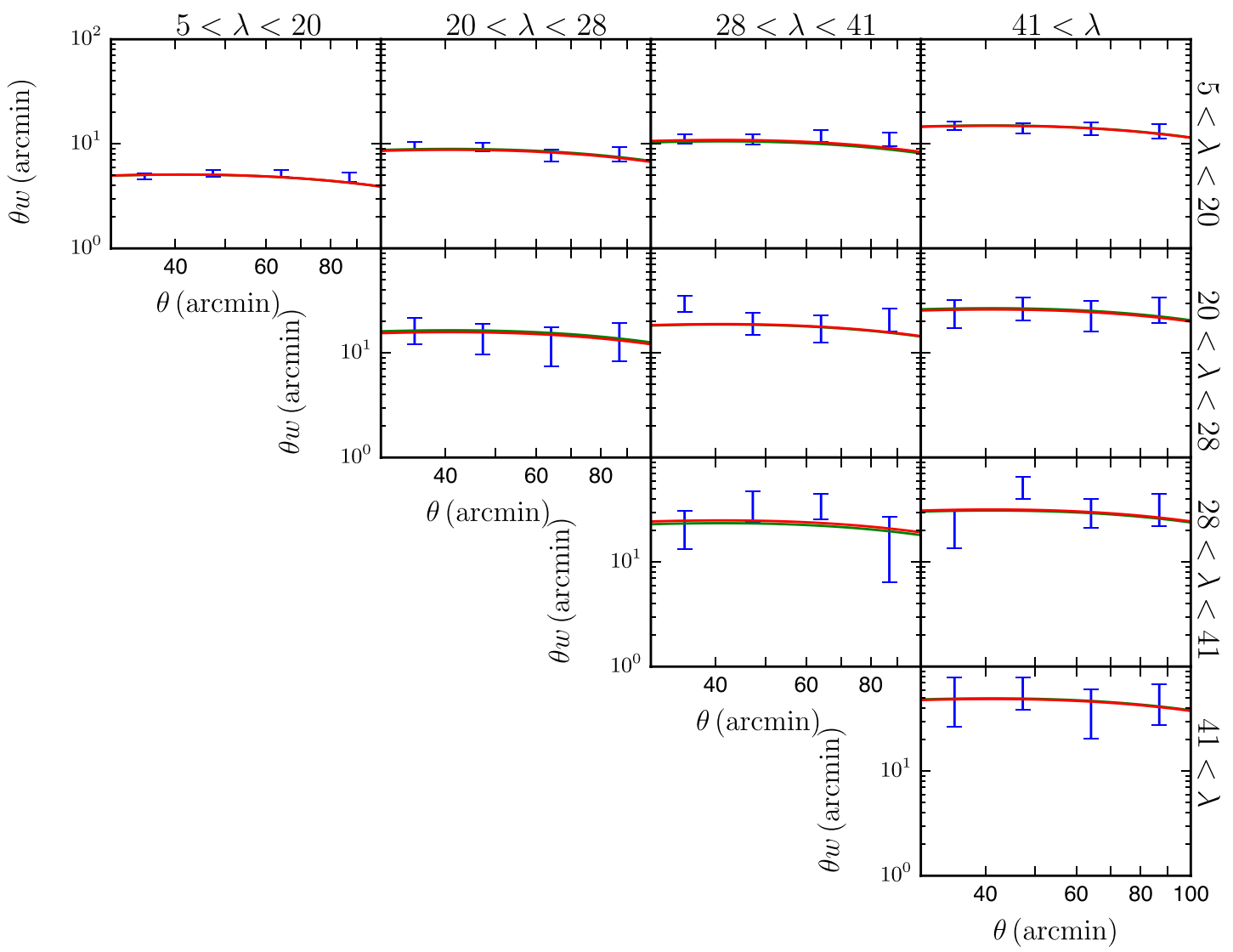

Figure A2. Same as Fig. A1, but for redMaPPer clusters with $0.26<z<0.33$.

This paper has been typeset from a $\mathrm{T}_{\mathrm{E}} \mathrm{X} / \mathrm{L} \mathrm{T}_{\mathrm{E}} \mathrm{X}$ file prepared by the author. 